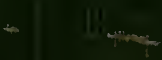




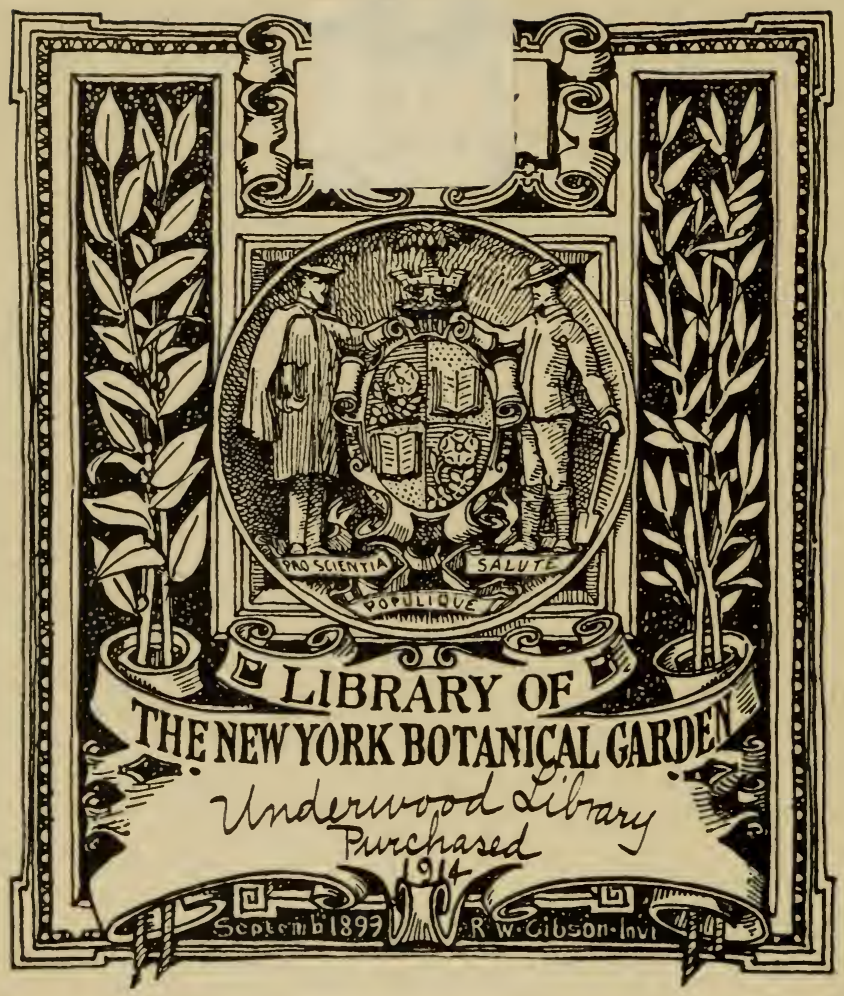






\section{MUSCI BOREALI-AMERICANI,}

Q U O R U M

SPECIMINA EXSICCATA

W. S. SULLIVANT ET L. LESQUEREUX

EDIDERUNT.

EDITIO SECUNDA.

COLUMBI OHIOENSIOM,

S U M P T I U S A U C TOR UM.

1865 . 


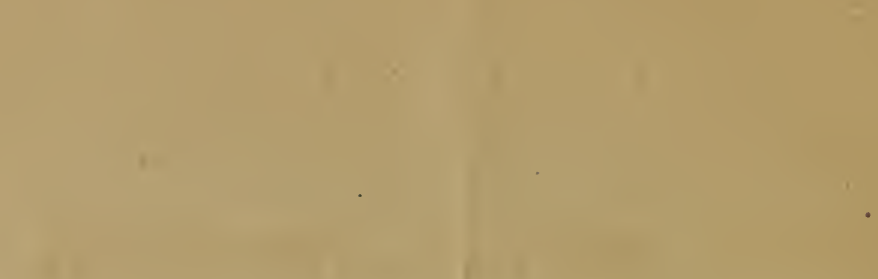

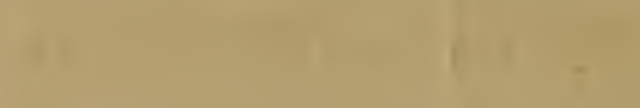




\section{MUSCI BOREALI-AMERICANI}

Q U O R U S

S P E C I I N A EXSICCA T A

\section{W. S. SULLIVANT et L. LESQUEREUX}

E D I D R U N T.

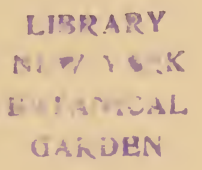

EDITIO SECUNDA.

COLUMBI OHIOENSIUM, S U M P T B U S A U T OR U M.

1865 . 
.591
1865

CANTABRIGIE:

TYPIS WELCH, BIGELOW, Et SOC., ACADEMIE TYPOGRAPHORCM. 


\section{MUSCI BOREALI-AMERICANI.}

1. Sphagnum macrophyllum.

Bernhardi. - Sulliv. Icon. Musc. p. 1, t. 1.

$\mathrm{H}_{\mathrm{AB}}$. in paludosis maritimis, a Nova Cæsarea usque ad Floridam; etiam per Montes Raccoon Alabamæ; frequens sed raro fertile.

2. Sphagnum Portoricense.

Hampe. - Sulliv. Icon. Musc. p. 3, t. 2.

S. Sullivantianum, Austin, in Amer. Jour. Sci. 1863, p. 252.

НАв. in turfosis Novæ-Cæsareæ (cl. C. F. Austin legit).

3. Sphagnum cymbifolium.

Ehrh. - Schimp. Gesch. d. Torfm. p. 69, t. 19.

$\mathrm{H}_{\mathrm{AB}}$. in turfosis vulgare.

4. Sphagnum molluscum.

Bruch. - Schimp. Gesch. d. Torfm. p. 69, t. 19.

$\mathrm{H}_{\mathrm{AB}}$. in turfosis Noræ-Cæsareæ (Austin). 
5. Sphagnum sedoides.

Brid. - Sulliv. Icon. Musc.p. 11, t. 6.

Haв. in rupibus irriguis summi scopuli Table Rock Carolinæ inferioris, et in montibus septentrionalibus.

6. Sphagnum Pylesit.

Brid. - Sulliv. Icon. Musc. p. 12, t. 6.

$\mathrm{H}_{\mathrm{AB}}$. in iisdem locis cum præcedente.

7. Sphagnum Pylesil, var.

HАв. in turfosis Noræ-Cæsareæ (Austin).

8. Sphagnum cyclophyllun.

Sulliv. \& Lesqx. - Sulliv. Icon. Musc. p. 13, t. 6.

$\mathrm{HAB}_{\mathrm{B}}$ in arenosis humidis meridionalibus.

9. Sphagnum squarrosum.

Pers. - Schimp. Gesch. d. Torfm. p. 63, t. 17.

$\mathrm{HAB}_{\mathrm{B}}$ in paludibus editioribus frequens.

10. Sphagnum cuspidatum.

Ehrh. - Schimp. Gesch.d. Torfm.p. 60, t. 16.

$\mathrm{HAB}_{\mathrm{A}}$, in stagnantibus meridionalibus.

11. Sphagnum cuspidatum, var.

S. Torreyanum, Sulliv. in Mem. Amer. Acad. n. s. 4, p. 175.

Haв. in Novæ Cæsareæ paludibus (cl. Torrey comm.). 


\section{Sphagnum cuspidatum, var.}

S. recurvum, Beauv. Prodr. p. 88.

$\mathrm{H}_{\mathrm{B}}$. in uliginosis a Nova-Anglia usque ad Georgiam.

\section{Sphagnum acutifolidir.}

Elırh. - Schimp. Gesch. d. Torfm.p. 57, t. 13, 14.

$\mathrm{H}_{\mathrm{AB}}$. in turfosis vulgare.

14. Sphagnum acutifoliun, pl. masc.

HAB. cum præcedente.

15. Sphagnum fimbriatum.

Wils. - Schimp. Gesch. d. Torfm.p. 59, t. 15.

HAв. in montibus Sierra Nevada Californiæ, alt. 11,000 ped. (cl. W. H. Brewer legit).

16. Sphagnum Rigidum.

Schimp. Gesch. d. Torfm. p. 65, t. 18.

НАв. in stagnantibus per montes Alabamæ.

17. Sphagnum Rigidum.

A. var. robustum. - B. var. simplex.

HAB. in iisdem locis cum No. 16.

18. Sphagnum humile.

Schimp. - Sulliv. Icon. Musc. p. 5, t. 3.

$\mathrm{HAB}_{\mathrm{B}}$ in sabulosis irriguis montium Lookout dict. Ala bamæ. 
19. Sphagnum Mulleri.

Schimp. Gesch. d. Torfm. p. 73, t. 26. - Sulliv. Icon. Musc. p. 9, t. 5 .

НАв. in arenosis irriguis Alabamæ.

\section{Sphagnum molle.}

Sulliv. Icon. Musc.p. 7, t. 4.

$\mathrm{H}_{\mathrm{AB}}$ ad rupes madidas cataractæ Tallulah Falls dictæ in Georgia; etiam in stagnantibus Novæ Cæsareæ.

\section{Sphagnum subsecundum.}

Nees. - Schimp. Gesch.d. Torfm.p. 74, t. 22, 23.

$\mathrm{HAB}_{\mathrm{B}}$ in turfosis profundis Ohionis superioris.

22. Sphagnum subsecundum, var.

S. Lescurii, Sulliv. Musc. Exsicc. ed. 1, No. 6.

$\mathrm{HAB}$, in stagnantibus meridionalibus fluitans.

23. Sphagnum auriculatum.

Schimp. Gesch. d. Torfm. p. 77, t. 24.

Forsan nonnisi varietas S. subsecundi.

HАв. per montes Sierra Nevada Californiæ, alt. 9,000 ped. (Brewer).

24. Andrat petrophila.

Ehrh. - Bryol. Europ. Andr. Monogr. p. 13, t. 1.

A. rupestris Hedw.

НАв. in Montibus Albis Noræ Angliæ (beat. Oakes legit). 
25. ANDREA RUPESTRIS.

Turn. - Bryol. Europ. Andr. Monogr. p. 21, t. 9 et 10. - A. Rothii, Web. et Mohr.

$\mathrm{H}_{\AA \text { B. }}$ ad rupes irriguas per montes Georgiæ et Carolinarum frequens.

26. ANdrea Crassinervia.

Bruch.-Bryol. Europ. Andr. Monogr. p. 23, t. 11.

НАв. in Noræ Angliæ montibus (beat. Oakes legit).

\section{Ephemeruir synoicum.}

James in Trans. Amer. Phil. Soc. (1865), p. 106.

Hermaphroditum, minutissimum, in prothallio cito evanescente nidulans; caule perbrevi, interdum longiore gracili ; foliis erecto-patentibus ecostatis, inferioribus ovato-lanceolatis, superioribus et perichætialibus longioribus lineari-lanceolatis integris vel apice subdenticulatis laxe oblongo-areolatis; capsula globosa solitaria (quandoque $2-3$ in eodem perichætio) subsessili ; calyptra minima ad stylidium solum basi paululum expansum reducta.

HАB. in fossis et agris argillosis prope Camden Noræ Cæsareæ (cl. T. P. James primus legit).

28. Ephemerum CRASSinervium.

Schwagr. - Sulliv. Icon. Musc. p. 17, t. 8.

$\mathrm{H}_{\mathrm{AB}}$. in agris argillosis Ohionis.

29. Ephemeruir coherens.

Hampe. - Bryol. Europ. Ephem. Monogr. p. 4, t. 1.

$\mathrm{H}_{\triangle \mathrm{B}}$. in terra humosa ad fluviorum ripas. 
30. Physcomitrella patens.

Bryol. Europ. Physcom. Monogr. p. 1, t. 2.-Phascum patens, Hedw.

$\mathrm{H}_{\mathrm{AB}}$. in argillosis humidis Ohionis.

31. Acaulon triquetrum, var.

C. Mull. - Bryol. Europ. Acaul. Monogr. p. 3, t. 1.

Pedicello breviore subcurvato.

$\mathrm{H}_{A B}$. in agris sabulosis siccis.

32. Acaulon muticum.

C. Mull. - Bryol. Europ. Acaul. Monogr. p. 3, t. 1 .

$\mathrm{H}_{\mathrm{AB}}$. in agris sabulosis Californiæ prope San Francisco (cl. H. N. Bolander, Californiæ muscorum indefessus indagator, legit et comm.).

\section{ACaulon Schimperianum.}

Sulliv. Icon. Musc. p. 18, t. 9.

$\mathrm{H}_{\mathrm{AB}}$. prope San Marcos in Texas (C. Wright); in republica Illinois (E. Hall).

34. Phascum cuspidatum.

Schreb. - Bryol. Europ. Phasc. Monogr.p. 4, t. 1.

$\mathrm{H}_{\mathrm{AB}}$. in pascuis humidis.

\section{Archidium Ohioense.}

Bryol. Europ. Mem. in Archid. Monogr. p. 3.-Sulliv. Icon. Musc. p. 16, t. 7.

HAB. in pratis humidiusculis, rarum. 
36. Pleuridium alternifolium.

Brid. - Bryol. Europ. Pleurid. Monogr. p. 3, t. 2.

$\mathrm{H}_{A B}$. in agris arenosis.

\section{Pleuridium alternifolium.}

Var. Lancastriense: foliis longioribus, areolatione densiore, capsula obtusiore, sporis majoribus. An species propria?

$\mathrm{H}_{\mathrm{AB}}$. in sabulosis Ohionis inferioris.

38. Pleuridium alternifolium.

Var. RoвUstum: statura majori, foliis perichætialibus brevioribus, sporis duplo majoribus.

$\mathrm{H}_{\mathrm{AB}}$. in arenosis humidis Raccoon montium Alabamæ.

39. Pleuridium subulatum.

Bryol. Europ. Pleurid. Monogr. p. 3, t. 1.

HAB. in arenosis Pennsylvaniæ et Californiæ (James et Bolander).

40. Pleuridium palustre.

Bryol. Europ. Pleurid. Monogr. p. 4, t. 2.

$\mathrm{H}_{\mathrm{AB}}$. in pratis humidis rarum (James comm.).

41. Bruchia flexuosa.

Schwagr. - Sulliv. Icon. Musc. p. 22, t. 13.

$\mathrm{HAB}_{\mathrm{A}}$ in argillosis humidis. 
42. Bruchia flexuosa.

Var. NIGRICANS : capsula subfusiformi, pedicello breviore, foliis brevioribus, sporisque majoribus.

$\mathrm{H}_{\mathrm{AB}}$. in jugis montium Raccoon Alabamæ.

43. Bruchia Ravenelit.

Wils. - Sulliv. Icon. Musc. p. 26, t. 16.

$\mathrm{H}_{\mathrm{AB}}$. in agris Carolinæ Inferioris (cl. H. W. Ravenel detexit).

44. Bruchia bRevifolia.

Sulliv. Icon. Musc. p. 25, t. 15.

$\mathrm{H}_{A B}$. in agris Texanis (Wright); in Carolina Inferiore (Ravenel legit).

45. BRUCHIA BREVIPES.

Hook. - Sulliv. Icon. Musc. p. 24, t. 14.

$\mathrm{H}_{A B}$. in arenosis humidis Carolinarum (Ravenel legit).

46. Astomum Sullivantit.

Bryol. Europ. - Sulliv. Icon. Musc. p. 20, t. 11.

НАв. ad margines sylvarum, frequens.

47. Astonum nitidulum.

Bryol. Europ. Mem. Astom. Monogr. p. 3.

$\mathrm{H}_{\mathrm{AB}}$. in pratis humidis, frequens. 
48. WEISIA CIRRHATA.

Hedw. - Bryol. Europ. Weis. Monogr. p. 9, t. 6.

$\mathrm{H}_{A B}$. in truncis præcipue deustis Californiæ, frequens (Bolander comm.).

49. WEISIA VIRIDULA.

Brid. - Bryol. Europ. Weis. Monogr. p. 5, t. 2.

НАв. in argillosis humidis vulgaris.

50. Grmnostomum RUPESTRe.

Schwagr.-Bryol. Europ. Gymnost. Monogr.p. 7, t. 5 et 6 .

HAB. ad rupes madidas per montes Pennsylvaniæ.

51. Grinostondur RUPESTRe, var.

Statura minore foliorumque areolatione densiore.

HAB. ad rupes arenaceas Ohionis.

52. Gymiostonum cURvirostrum.

Hedw. - Bryol. Europ. Gymnost. Monogr. p. 8, t. 7 et 8 .

$\mathrm{H}_{\mathrm{AB}}$ ad rupes irriguas frequens.

\section{Gymnostonum CURVirostrum, var.}

Capsula ovato-cylindrica fusco-lutea.

$\mathrm{H}_{\mathrm{AB}}$. in rupibus irriguis Niagarce cataractæ. 
54. Hymenostomum microstonum.

R. Brown. - Bryol. Europ. Hymen. Monogr. p. 4, t. 1. $\mathrm{H}_{A B}$. ad terram argillaceam Ohionis occidentalis; etiam in agris Texanis (legit Wright).

55. Rhabdoweista fugax.

Bryol. Europ. Rhab. Monogr. p. 4, t. 1.

НАв. in Novæ-Angliæ montibus (Oakes comm.).

56. Rhabdoweisia denticulata.

Bryol. Europ. Rhab. Monogr. p. 5, t. 2.

Нав. in Novæ-Angliæ Montibus Albis (Oakes); etiam in summo cacumine Black montis Carolinæ Sept.

57. Dicranum gracilescens.

Web. et Mohr.-Bryol. Europ. Dicr. Monogr.p. 13, t.1. $\mathrm{H}_{\mathrm{AB}}$. in Novæ Angliæ montibus (Oakes).

58. Dicranum polycarpum.

Ehrh. - Bryol. Europ. Dicr. Monogr. p. 14, t. 2.

НАв. ad rupes Lacus Superioris (cl. Agassiz comm.).

59. Dicranum virens.

Hedw. - Bryol. Europ. Dicr. Monogr. p. 15, t. 3.

$\mathrm{H}_{\mathrm{AB}}$. ad arborum truncos putridos in pinetis editoribus.

60. Dicranum pellucidum?

Hedw. - Bryol. Europ. Dicr. Monogr. p. 16, t. 4.

A forma normali statura minore, foliis siccitate incur- 
rato-crispatis humiditate erectis subapertis (nec reflexis) brevioribus lanceolatis acutis, areolatione densiore basim versus angustiore, capsulaque longiore differt. An species propria?

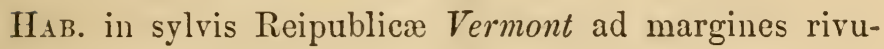
lorum (Frost).

\section{Dicranum Schrebert.}

Hedw. - Bryol. Europ. Dicr. Monogr. p. 18, t. 6. $\mathrm{H}_{A B}$. in montibus editoribus Noræ Angliæ, sterile (James); et in montibus Adirondack fertile, e rarioribus.

62. Dicranum Debile.

Hook. et Wils. - Sulliv. Icon. Musc. p. 33, t. 20. $\mathrm{H}_{\mathrm{AB}}$. in solo argilloso Alabamæ inferioris.

\section{Dicranum vartum.}

Hedw. - Bryol. Europ. Dicr. Monogr. p. 20, t. 10 et 11.

НАв. in argillosis præruptis humidis.

64. Dicranum rufescens.

Turn. - Bryol. Europ. Monogr. p. 22, t. 12.

Har. per montes Pennsylvaniæ in argillosis humidis.

65. Dicranum cerviculatum.

Hedw. - Bryol. Europ. Dicr. Monogr. p. 22, t. 9. Нав. in Novæ Cæsareæ turfosis (cl. Torrey comm.). 
66. Dicranum subulatum.

Hedw. - Bryol. Europ. Dicr. Monogr. p. 23, t. 13.

Hab. in montibus Novæ Angliæ (Oakes).

67. Dićranum heteromallum.

Hedw. - Bryol. Europ. Dicr. Monogr. p. 25, t. 15.

HAB. ad terram rupesque madidas.

68. Dicranum heteromallum.

Var. orthocarpuir, C. Mull. Synop. 1, p. 433.

$\mathrm{H}_{\triangle \mathrm{B}}$. ad rupes arenaceas irriguas Ohionis.

69. Dicranum Blyttii.

Bryol. Europ. Dicr. Monogr. p. 26, t. 16.

Hab. in Novæ Angliæ Montibus Albis (Oakes).

70. Dicranum Starkit.

Web. et Mohr. - Bryol. Europ. Dicr. Monogr. p. 27, t. 17.

Haв. in Novæ Angliæ montibus (beat. Oakes).

71. Dicranum montanum.

Hedw. - Bryol. Europ. Dicr. Monogr. p. 29, t. 20.

$\mathrm{H}_{\mathrm{AB}}$. in truncis emortuis prope Niagarce cataractam; etiam per montes Novæboracenses sat frequens.

72. Dicranum flagellare.

Hedw. - Bryol. Europ. Dicr. Monogr. p. 30, t. 21.

$\mathrm{H}_{\mathrm{AB}}$. in sylvis, ad truncos putridos vulgare. 
73. Dicranum interruptum.

Bryol. Europ. Dicr. Monogr. p. 30, t. 22.

$\mathrm{H}_{\mathrm{AB}}$. ad rupes arenaceas in umbrosis montanis.

\section{Dicranum longifolium.}

Hedw. - Bryol. Europ. Dicr. Monogr. p. 32, t. 25.

HAB. ad rupes et arbores in montibus editioribus.

\section{Dicranum scoparium.}

Hedw. - Bryol. Europ. Dicr. Monogr. p. 34, t. 16 et 17.

$\mathrm{H}_{A B}$ ad radices arborum in sylvis montanis.

\section{Dicranum scoparium, var.}

Foliis latioribus squarroso-reflexis ; perichætialibus costatis apice longius acuminatis flexuosis dentatis; capsula angustiore, et cæt.

HАв. ad rupes graniticas Pennsylvaniæ montosæ.

- 77. Dicranum scopariun, var.

Foliis angustioribus, cellulis basi haud sinuosis, costa dorso minus alata; capsula pedicelloque pallidioribus.

Inflorescentia (revera dioica) interdum, ob gemmulas masculas inque tomento radiculari nidulantes, pseudo-monoica videtur.

D. scoparium, Musc. Alleghan. n. 155. - D. pallidum, Mull. Synop. Musc. 1, p. 359, nec Bryol. Europ.

$\mathrm{H}_{\mathrm{AB}}$. in sylvis, ad terram lignaque putrida, montosa fugiens. 
78. Dicranum scopariun, var.

Capsula ovato-cylindrica breviore.

$\mathrm{HAB}_{\mathrm{B}}$, in sylvis montanis.

\section{Dicranum elongatum.}

Schwagr. - Bryol. Europ. Dicr. Monogr. p. 35, t. 28. НАв. in turfosis per montes Novæ Angliæ (beat. Oakes legit).

80. Dicranum congestum.

Brid. - Bryol. Europ. Dicr. Monogr. p. 36, t. 29.

НАв. in sylvis editoribus ad truncos emortuos.

\section{Dicranum Muhlenbeckit.}

Bryol. Europ. Dicr. Monogr. p. 38, t. 30.

Нав. prope Santa Fé Neo-Mexicanum (A. Fendler legit).

\section{Dicranum rhabdocarpun.}

Sulliv. Mem. Amer. Acad. of Arts \& Sci. ser. 2, 4, $1849, p .173, t .3$.

НАв. in pinetis subalpinis Montium Rupestrium ( $E$. Hall legit.)

83. Dicranum palustre.

Brid. - Bryol. Europ. Dicr. Monogr. p. 39, t. 31. Var. foliis grossius serratis, costaque dorso versus apicem dentata.

НАв. in turfosis profundis Ohionis et Pennsylvaniæ. 


\section{Dicranum Schraderi.}

Schwagr. - Bryol. Europ. Dicr. Monogr. p. 40, t. 32. $\mathrm{H}_{\mathrm{AB}}$. in turfosis sylvisque humidis frequens.

\section{Dicranum spurium.}

Hedw. - Bryol. Europ. Dicr. Monogr. p. 41, t. 33.

Var. foliis dorso minus papillosis.

D. pallidum, Bryol. Europ. Mem. l. c. p. 39.

НАв. in arenosis frequens.

86. Dicranum spurium, var.

Hedw.-D. condensatum, Hedw. Sp. Musc. p. 139, t. 34 .

$\mathrm{H}_{\mathrm{AB}}$. in arenosis siccis meridionalibus.

87. Dicranum undulatum.

Turn. - Bryol. Europ. Dicr. Monogr. p. 42, t. 34 et 35 .

$\mathrm{HAB}_{\mathrm{B}}$ in sylvis editioribus.

88. Dicranum Drummondi.

C. Mull. - D. robustum, Bryol. Europ. Dicr. Monogr. p. $43, t .36$.

HAB. in sylvis ad Lacus Superioris litora septentrionalia (Agassiz) : etiam in Novæ Angliæ montibus.

89. Arctoa fulvella.

Bryol. Europ. Arct. Monogr. p. 4, t. 1. Haв. in Novæ Angliæ Montibus Albis (Oakes). 
90. Dicranodontium longirostre.

Web. et. Mohr. - Bryol. Europ. Dicranod. Monogr. p. $2, t .1$.

$\mathrm{H}_{\mathrm{AB}}$. in rupium fissuris et ad terram arenosam Ohionis inferioris, sterile.

\section{Campylopus Viridis.}

Sulliv. \& Lesqx. - Sulliv. Icon. Musc. p. 30, t. 18.

$\mathrm{H}_{\mathrm{AB}}$. in sylvis ad truncos putridos, a Nora-Anglia usque ad Ohionem, haud rarum, semper sterile.

92. Campylopus leucotrichus.

Sulliv. \& Lesqx. - Sulliv. Icon. Musc. p. 28, t. 17.

$\mathrm{H}_{\mathrm{AB}}$. ad rupes in sabulosis siccis per Raccoon montes Alabamæ.

93. Campylopus Tallulensis.

Sulliv. \& Lesqx. - Sulliv. Icon. Musc. p. 27, t. 17.

$\mathrm{H}_{A B}$. ad rupes irriguas cataractæ Tallulah Falls dictæ, in Georgia montosa.

\section{Camipylopus Leanus.}

Sulliv. Icon. Musc. p. 29, t. 18.

$\mathrm{H}_{\mathrm{AB}}$. in sylvis ad truncos putridos sat frequens, semper sterile.

95. Trematodon longicollis.

Michx. - Sulliv. Icon. Musc. p. 31, t. 19.

$\mathrm{H}_{\mathrm{AB}}$. in arenosis humidis meridionalibus. 
96. Trematodon ambiguUs.

Hedw. - Bryol. Europ. Tremat. Monogr. p. 5, t. 2.

$\mathrm{H}_{\mathrm{AB}}$. in arenosis sylvaticis vel montanis, septentrionalia deligens.

\section{Leucobryum vulgare.}

Hampe.-Oncophorus glaucus, Bryol. Europ. Onc. Monogr.p. 5, t. 1 et 2.

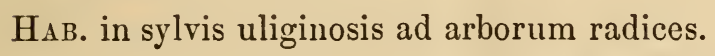

(Matur. Decembri.)

98. LeUCobryum Minus.

Hampe in Linnca, 18, p. 42.

$\mathrm{H}_{\mathrm{AB}}$, in siccioribus Olionis septentrionalis.

(Matur. Junio.)

99. Fissidens овтusifolius.

Wils. - Sulliv. Icon. Musc. p. 35, t. 22.

$\mathrm{H}_{\mathrm{AB}}$. ad lapides arenosas in rivulis.

100. Fissidens exiguus.

Sulliv. Icon. Musc. p. 36, t. 23.

$\mathrm{H}_{\mathrm{AB}}$ ad lapides rupesque irriguas.

101. Fissidens minutulus.

Sulliv. Icon. Musc. p. 37, t. 24.

НАв. in iisdem locis cum præcedente, rarius. 
102. Fissidens Ravenelii.

Sulliv. Icon. Musc. p. 39, t. 25.

HAB. ad margines fossarum circa Carlopolim Carolinæ Inferioris (cl. Ravenel detexit).

103. Fissidens Bryoides.

Hedw. - Bryol. Europ. Fiss. Monogr. p. $\dot{8}, t .2$.

$\mathrm{HAB}_{\mathrm{A}}$. in præruptis umbrosis sylvarum.

104. Fissidens incurvus.

Schwagr._Bryol. Europ. Fiss. Monogr. p. 6, t. 1.

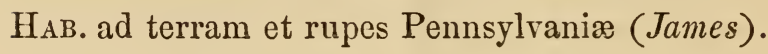

105. Fissidens linibatus.

Sulliv. in Bot. Pacif. R. R. Surv. (Whipple), p. 185, t.1. $\mathrm{H}_{\mathrm{AB}}$, ad terram umbrosam in sylvis et graminosis Californiæ (Bolander comm.).

106. Fissidens taxifolius.

Hedw. - Bryol. Europ. Fiss. Monogr. p. 9, t. 4.

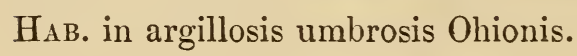

107. Fissidens subbasilaris.

Hedw. - Sulliv. Icon. Musc. p. 41, t. 26.

$\mathrm{H}_{\mathrm{AB}}$. in sylvis ad arborum radices.

108. Fissidens adiantoides.

Hedw. - Bryol. Europ. Fiss. Monogr. p. 10, t. 5.

НАв. in humidis umbrosis ad terram et rupes. 
109. Fissidens osmundioides.

Hedw. - Bryol. Europ. Fiss. Monogr. p. 8, t. 3.

$\mathrm{H}_{\mathrm{AB}}$. ad terram arborumque radices, rarius.

110. Fissidens polypodioides.

Hedw. - Sulliv. Icon. Musc. p. 42, t. 27.

НАВ. ad rupes irriguas Georgiæ superioris, sterile.

111. Fissidens grandifrons.

Brid. - Bryol. Europ. Fiss. Monogr. p. 11, t. 6.

$\mathrm{H}_{\mathrm{AB}}$. in rupibus irroratis ad Niagare cataractam, sterilis.

112. Cononitriuni Julianun. •

Mont. - Octodiceras Julianum, Bryol. Europ. Octod. Monogr.p. 4, t. 1.

НАв. in lapidibus rivulorum ab Ohione ad Tennessee, rarius.

113. Blindia acuta.

Bryol. Europ. Blind. Monogr. p. 3, t. 1.

Haв. ad rupes irriguas montium Catskill Reipublice Neo-Eboracensis.

\section{Campylosteliun saxicola.}

Bryol. Europ. Campyl. Monogr. p. 3, t. 1.

Hab. in rupibus erraticis Noræ Anglixe (cl. R. C. Ingraham) ; etiam ad rupes arenaceas Reipublice Kentucky, rariss. 
115. Pottia Heinis.

Bryol. Europ. Pott. Monogr. p. 12, t. 7.

HAB. ad terram in alpinis Montium-Rupestrium (Hall).

116. Pottia truncata.

Hedw. - Bryol. Europ. Pott. Monogr. p. 9, t. 4 et 5. НАв. in agris cultis Novæ Angliæ.

117. Ротtia minutula.

Schwegr. - Bryol. Europ. Pott. Monogr. p. 8, t. 3. НАв. in agris Texanis prope San Marcos (C. Wright).

118. Pottia subsessilis.

Schwcegr. - Bryol. Europ. Pott. Monogr. p. 6, t. 1.

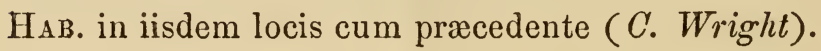

119. Anacalypta Starkeana.

Hedw. - Bryol. Europ. Anac. Monogr. p. 2, t. 1.

НАв. in terra argillosa Californiæ (Bolander).

120. Desmatodon arenaceus.

Sulliv. \& Lesqx. - Sulliv. Icon. Musc. p. 45, t. 29.

HAB. ad rupes arenaceas circa Lancaster Ohionis.

121. Desmatodon nervosus.

Bryol. Europ. Desmat. Monogr.p. 6, t. 3.

Et var. $\beta$. edentulus $l$. $c$.

$\mathrm{HAB}_{\text {a }}$ in aggeribus limosis et muris argillaceis Californiæ (Bolander). 
122. Desmatodon latifolius.

Brid. - Bryol. Europ. Desmat. Monogr. p. 5, t. 1.

$\mathrm{HAB}_{\mathrm{A}}$ ad terram in editoribus Montium-Rupestrium (Hall); in montibus Californiæ (Brewer).

123. Desmatodon plinthobius.

Sulliv. \& Lesqx. - Sulliv. Icon. Musc. p. 47, t. 30.

HAB. ad pavimenta lateritia Carlopolis Carolinæ Inferioris (Ravenel); etiam in Republica Tennessee ad Nashville collegii muros.

124. Desmatodon Neo-Mexicanus.

Sulliv. \& Lesqx. - Sulliv. Musc. exsicc. Ed.1, No. 95. НАв. in agris Neo-Mexicanis (legit C. Wright).

125. BARBULA RIGIDA.

Schultz. - Bryol. Europ. Barbul. Monogr. p. 13, t.1. $\mathrm{H}_{\mathrm{AB}}$. in pascuis aridis Reipublicæ Illinois, sterile ( Hall).

126. Barbula membranifolia.

Schultz. - Bryol. Europ. Barb. Monogr.p. 17, t. 3.

НАв. ad rupes in California (legit Bigelow).

\section{Barbula papillosa.}

Wils. - Bryol. Brit. p. 135, t. 44.

$\mathrm{HAB}_{\mathrm{B}}$ ad arborum præsertim ulmorum corticem, in locis apertis Noræ Angliæ. 
128. Barbula unguiculata. Hedw. - Bryol. Europ. Barb. Monogr. p. 18, t. 5 et 6. $\mathrm{HAB}_{\mathrm{B}}$ ad terram et muros ubique.

129. Barbula FAllax.

Var. BRevicaulis, Bryol. Europ. Barbul. Monogr. p. 23, t. 9 .

НАв. in montibus Reipublicæ Vermont (Frost).

130. Barbula vinealis.

Brid. - Bryol. Europ. Barb. Monogr. p. 24, t. 10.

НАв. ad rupes madidas Californiæ (Bigelow, Bolander).

131. Barbula viNealis.

Var. Flaccida. Bryol. Europ. $l$. $c$.

$\mathrm{H}_{\text {A }}$. in iisdem locis ac præcedens (Bolander).

132. Barbula Flexifolia.

Hampe in Linnæa, (1859,) p. 456.

НАв. in arenosis Californiæ perfrequens (Bolander).

133. Barbula artocarpa.

Lesqx. in Trans. Amer. Phil. Soc. 13, p. 4.

HAв. ad terram in monte Diablo prope San Francisco Californiæ (Bolander legit).

134. Barbula virescens.

Lesqx. in Trans. Amer. Phil. Soc. 13, p. 4.

HAB. ad rupes erraticas secus littora Californiæ sinûs (Bolander). 
135. Barbula BRACHYPhylla.

Sulliv. in Bot. Pacif. R. R. Survey (Whipple) p. 186, t. 2.

$\mathrm{H}_{\mathrm{AB}}$. in California (legit Bigelow).

136. Barbula Caspitosa.

Schwagr. - B. cirrhata, Bryol. Europ. Barb. Monogr. p. $24, t .11$.

HAB. ad terram arborumque radices.

137. Barbula tortuosa.

Web. et Mohr.-Bryol. Europ. Barb. Monogr. p. 26, t. 13.

HАв. ad rupes calcarias insulæ Goat Island dictæ, juxta cataractam Niagara.

138. Barbula convoluta.

Hedw. - Bryol. Europ. Barb. Monogr. p. 29, t. 16.

$\mathrm{H}_{\mathrm{AB}}$. in montosis siccioribus ad terram.

139. Barbula bolaNderi.

Lesqx. in Trans. Amer. Plil. Soc. 13, p. 5.

$\mathrm{H}_{\mathrm{AB}}$. in rupibus Californiæ (Bolander).

140. Barbula anplexa.

Lesqx. in Trans. Amer. Phil. Soc. 13, p. 5.

$\mathrm{HAB}_{\mathrm{A}}$ in scaturiginosis Californiæ (Bolander). 
141. Barbula muCronifolia.

Schwagr. - Bryol. Europ. Barb. Manogr. p. 38, t. 23. $\mathrm{H}_{\mathrm{AB}}$. ad rupes calcarias humo tectas.

142. Barbula inermis.

Mont. - Bryol. Europ. Barb. Monogr. Suppl. 3, t. 3. $\mathrm{H}_{\mathrm{AB}}$. ad rupes secus flumen Colorado Californiæ (legit Bigelow).

143. Barbula latipila.

Bryol. Europ. Barb. Monogr. p. 40, t. 25.

Var. partibus omnibus multo minoribus et gracilioribus.

HAB. ad terram in Monte Diablo Californiæ (Bolander).

\section{Barbula latifolia.}

Bryol. Europ. Barb. Monogr. p. 41, t. 24.

$\mathrm{H}_{\mathrm{AB}}$. in ligno subimmerso rivulorum Californiæ, rariss. (Bolander detexit).

145. Barbula RURALis.

Hedw. - Bryol. Europ. Barb. Monogr. p. 42, t. 27.

$\mathrm{H}_{A B}$. in agris Texanis (legit Wright).

146. BarbUla RURALIS, var.

Caule elongato; foliis e basi squarroso reflexis, areolatione papillosa, papillis elongatis furcatis, costa dorso spinuloso-rugosa.

$\mathrm{H}_{\mathrm{AB}}$. ad rupes umbrosas Californiæ (Bolander). 
147. Barbula Mulleri.

Bryol. Europ. Barb. Monogr. p. 44, t. 28.

HAB. ad truncos terramque Californiæ, perfrequens (Bigelow, Bolander).

\section{Trichostonum flexipes.}

Bryol. Europ. Trichost. Monogr. p. 6, t. 2.

HAB. in California, ad truncos putridos terramque umbrosam (Bigelow, Bolander).

149. Trichostomum Fragile.

Hook. in Drumm. Musc. Amer. Coll. 1, No. 127.

HAB. ad Lacus Superioris rupes, sterile (cl. Agassiz); etiam ad rupes fluminis $A u$ Sable Noræboracensis.

150. Trichostonum Rigidulun.

Smith. - Bryol. Europ. Trichost. Monogr. p. 10, t. 7. HAB. ad Niagare fluminis rupes humidas.

151. Trichostonum tophaceum.

Brid. - Bryol. Europ. Trichost. Monogr. p. 9, t. 6. $\mathrm{H}_{\mathrm{AB}}$. in rupibus tophaceis irroratis Californiæ (Bolander legit).

152. Trichostomum tortile.

Schrad. - Bryol. Europ. Trichost. Monogr. p. 14, t. 10. $\mathrm{H}_{\mathrm{AB}}$. in solo argilloso ad margines viarum. 
153. Trichostomum tortile.

Var. pusillum, Bryol. Europ. l.c.

$\mathrm{H}_{A B}$. ad margines viarum Pennsylvaniæ (James).

154. Trichostonum vaginans.

Sulliv. Icon. Musc. p. 43, t. 28.

НАв. in terra argillacea in montosis.

155. Trichostonum palliduir.

Hedw. - Bryol. Europ. Trichost. Monogr. p. 18, t. 14. $\mathrm{HAB}_{\mathrm{A}}$ in arenosis et argillosis frequens.

156. Trichostonum Glaucescens.

Hedw. - Bryol. Europ. Trichost. Monogr. p. 18, t. 15. $\mathrm{H}_{A B}$. in montibus Vermont (Frost legit).

157. Didymodon RUbellús.

Bryol. Europ. Didym. Monogr. p. 3, t.1.

HAB. ad terram et rupes humidas.

158. Didymodon cylindricus.

Nees et Hornsch. - Bryol. Europ. Didym. Monogr. p. $5, t .3$.

Нав. in truncis deciduis Pennsylvaniæ (legit James).

159. Ceratodon purpureus.

Brid.-Bryol. Europ. Cerat. Monogr. p. 5, t. 1 et 2. $\mathrm{H}_{\mathrm{AB}}$. in truncis putridis terraque humida ubique. 
160. Ceratodon purpureus.

Var. XANTHOPUS.

HAB. in truncis putridis Californiæ (Bigelow, Bolander).

\section{Distichium Capillaceum.}

Bryol. Europ. Distich. Monogr. p. 4, t. 1.

HАв. in alpinis Noræ Angliæ (Oakes); ad rupes Lacus Superioris (cl. Agassiz).

162. Distichiuni inclinatum.

Bryol. Europ. Distich. Monogr. p. 5, t. 2.

НАв. in iisdem locis cum præcedente.

163. Eustichiun Norvegicun.

Bryol. Europ. - Phyllogonium Norvegicum, Brid. Sulliv. in Mem. Amer. Acad. n. s. 3, p. 57, t. 1.

НАв. ad rupes arenaceas in umbrosis et humidis Ohionis inferioris; etiam in Kentucky meridionali; semper sterile.

164. Tetraphis pellucida.

Hedw. - Bryol. Europ. Tetraph. Monogr. p. 6, t. 1.

$\mathrm{H}_{\mathrm{AB}}$. in sylvaticis ad truncos putridos.

165. Encalypta ciliata.

Hedw. - Bryol. Europ. Encalypt. Monogr. p. 10, t. 3.

$\mathrm{HAB}_{\mathrm{A}}$ in montibus editoribus, ad rupes humidas. 
166. Encalypta rhabdocarpa.

Schwagr.-Bryol. Europ. Encalypt. Monogr.p. 13, t. 6.

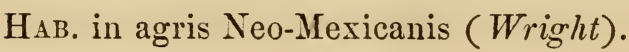

\section{Excalypta vulgaris.}

Hedw. - Bryol. Europ. Encalypt. Monogr. p. 9, t. 2.

Haв. per montes Californiæ (Bolander); etiam in Montibus Rupestribus (Hall).

168. Syrropodon Floridanus.

Sulliv. Icon. Musc. p. 49, t. 31.

HАв. in Florida (legit cl. Chapman).

169. Zygodon Sullivantir.

C. Mull. - Sulliv. Icon. Musc. p. 51, t. 32.

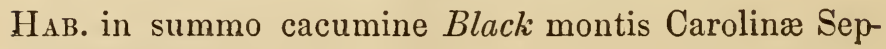
tentrionalis, sterile.

170. Zygodon Mougeoti.

Bryol. Europ. Zygod. Monogr.p. 7, t. 1.

$\mathrm{H}_{\mathrm{AB}}$. in truncis arborum Pennsylvaniæ, sterile (James).

171. Zygodon Lapponicus.

Hedw. - Bryol. Europ. Zygod. Monogr. p. 6, t. 1. $\mathrm{H}_{\mathrm{AB}}$. ad rupes in montanis editioribus.

\section{Zygodon Californicus.}

Hampe in Bot. Zeit. 1862, p. 361.

$\mathrm{H}_{\mathrm{AB}}$. in rupibus umbrosis Californiæ (Bolander). 
173. ORthotrichum OBTUSIFOLIUM.

Schrad. - Bryol. Europ. Orthot. Monogr. p. 13, t. 1. HAв. ad salices circa stagnum Fresh Pond dictum, prope Cantabrigiam in Nova Anglia.

\section{ORthotrichum ROGERI?}

Brid., nec Bryol. Europ.

$\mathrm{H}_{\mathrm{AB}}$, in cortice populorum Pennsylvaniæ et Novæboracensis.

\section{Orthotrichum Sturmil.}

Hornsch. et Hoppe. - Bryol. Europ. Orthot. Monogr. p. 9, t. 2.

Hab. prope Santa Fé Neo-Mexicanorum (Wright); in Novæ Angliæ alpibus (Oakes).

176. Orthotrichum cupulatum.

Hoffm. - Bryol. Europ. Orthot. Monogr. p. 8, t. 2.

$\mathrm{H}_{\mathrm{AB}}$. ad rupes calcarias prope Nashville in Tennessee.

177. Orthotrichum anomalum.

Hedw. - Bryol. Europ. Orthot. Monogr. p. 10, t. 3.

$\mathrm{H}_{\mathrm{AB}}$. ad rupes Niagara cataractæ (Agassiz).

178. Orthotrichum CYLINDRoCARPUM.

Lesqx. in Trans. Amer. Phil. Soc. 13, p. 6.

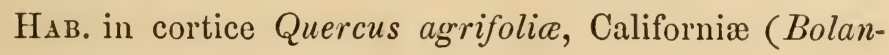
der legit). 
179. Orthotrichum strangulatum.

Beauv. - Sulliv. Icon. Musc. p. 57, t. 36.

$\mathrm{HAB}_{\mathrm{B}}$ in arboribus frequens.

180. Orthotrichum PSilocarpun.

James in Trans. Amer. Phil. Soc. 13, p. 110.

$\mathrm{H}_{\mathrm{AB}}$. in cortice arborum prope Philadelphiam Penn. (James comm.).

181. Orthotrichum Canadense.

Br. et Sch. in Lond. Journ. Bot. 1843, p. 667.

$\mathrm{H}_{A B}$. cum priori, sed rarius invenitur.

182. Orthotrichum afFine.

Schrad.-Bryol. Europ. Orthot. Monogr. p. 17, t. 7.

$\mathrm{H}_{\mathrm{AB}}$. in arboribus prope Lacum Superiorem (Agassiz).

183. ORTHOTRICHUM LEIOCARPUM.

Bryol. Europ. Orthot. Monogr. p. 28, t. 15.

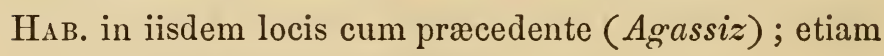
per montes Novæboracenses.

184. ORthotrichum Lyellit.

Hook. - Bryol. Europ. Orthot. Monogr.p. 27, t. 16.

НАв. in sylvis Californiæ ad arbores, frequens (Bolander comm.). 
185. Orthotrichum Lyelli, var.

Foliis eorumque papillis longioribus.

O. papillosum, Hampe in Linnaa (1859) p. 458.

НАв. cum præcedente (Bolander comm.).

186. Orthotrichum SPECiosum.

Nees. - Bryol. Europ. Orthot. Monogr. p. 19, t. 9.

НАв. in arboribus sylvarum tractus inferioris Montium Rupestrium (Hall legit).

187. Orthotritchum Texanum.

Sulliv. Icon. Musc. p. 53, t. 34.

НАв. in agris Texanis (Wright).

188. Orthotrichum Hutchinsiæ.

Hook. et Tayl. - Bryol. Europ. Orthot. Monogr. p. 20, t. 10 .

НАв. in rupibus graniticis per montes frequens.

189. Orthotrichum LUdwigir.

Schwagr. - Bryol. Europ. Orthot. Monogr. p. 12, t. 4. $\mathrm{HAB}_{\mathrm{A}}$ in sylvis montanis ad arbores.

190. Orthotrichum CRISPUM.

Hedw. - Bryol. Europ. Orthot. Monogr. p. 2:3, t. 12.

$\mathrm{H}_{\mathrm{AB}}$. in montibus editioribus, arboreum. 
191. Orthotrichum CRispulum.

Hornsch. - Bryol. Europ. Orthot. Monogr. p. 23, t.12.

$\mathrm{H}_{A B}$, ad truncos arborum, præcipue fagorum, haud rarum.

192. Schlotheimia SullivantiI.

C. Mull. - Sulliv. Icon. Musc. p. 61, t. 38.

$\mathrm{H}_{A B}$ ad rupes arboresque in montibus Georgix; etiam juxta Novam-Aureliam Louisianæ.

\section{Macromitrium Dregei.}

Hornsch. - Sulliv. Icon. Musc. p. 59, t. 37.

$\mathrm{H}_{\mathrm{AB}}$. in summo cacumine Yonah montis Georgix, ad pinorum corticem.

\section{Drummondia clavellata.}

Hook. - Sulliv. Icon. Musc. p. 52, t. 33.

$\mathrm{H}_{A B}$. ad corticem arborum sat frequens.

195. Pтychomitrium incurvum.

Schwagr. - Sulliv. Icon. Musc. p. 63, t. 39.

$\mathrm{H}_{\mathrm{AB}}$. ad rupes in umbrosis montanis.

196. Ptychomitrium Drummond.

Hook. \& Wils. - Sulliv. Icon. Musc. p. 65, t. 40.

$\mathrm{H}_{\mathrm{AB}}$. ad truncos arborum in Carolina Inferiore (legit Ravenel). 
197. Coscinodon Wrightil.

Sulliv. Icon. Musc. p. 71, t. 45.

Hab. juxta Santa Fé Neo-Mexicanorum (Wright legit).

198. Schistidium APOCARPUn.

Hedw. - Bryol. Europ. Schistid. Monogr. p. 7, t. 3 et 4. НАв. ad rupes murosque vulgare.

199. Schistidium apocarpum.

Var. Gracile, Bryol. Europ. l. c.

НАв. in summis montibus Georgiæ, ad rupes.

200. Schistidiun APocarpum.

Var. rivulare, Bryol. Europ. l. c.

НАB. in rupibus irriguis rivulorum, rarum.

201. SChistidium CONFERTUM.

Funk. - Bryol. Europ. Schistid. Monogr. p. 7, t. 2.

HAB. per montes Alleghanienses, rupestre.

202. SCHISTIDIUM CONFERTUM.

Var. obtusifolium, Bryol. Europ. l. $c$.

Haв. in alpinis Noræ Angliæ (Oakes).

203. Schistidium AgassiziI.

Sulliv. \& Lesqx. - Sulliv. Mosses U. States, p. 104.

Hab. ad rupes irriguas Lacus Superioris (cl. Agassiz legit). 
204. Grimima plagiopodia.

Hedw. - Bryol. Europ. Grimm. Monogr. p. 9, t. 1.

$\mathrm{HAB}_{\mathrm{AB}}$ in alpinis per Montes Rupestres (Hall legit).

205. Grimmia Californica.

Sulliv. in Bot. Pacif. R. R. Survey (Whipple) p. 187, t. 4.

$\mathrm{H}_{\mathrm{AB}}$. in rupibus umbrosis Californiæ (Bolander comm.).

206. Grimmia Californica, var.

Capsula subglobosa, operculo longiori.

$\mathrm{H}_{\mathrm{AB}}$. in iisdem locis ac præcedens.

207. Grimmia trichophylla.

Grev. - Bryol. Europ. Grimm. Monogr. p. 16, t. 9.

$\mathrm{H}_{\mathrm{AB}}$. in rupibus erraticis umbrosis Californix (Bolander legit).

208. Grimmia trichophylla, var.

Colore atro-virente, pilo foliorum breviori, etc.

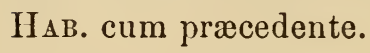

209. Grimmia Olneyi.

Sulliv. Icon. Musc. p. 67, t. 42.

НАв. in rupibus maritimis Rhodensis Insulæ (cl. S. T. Olney legit).

210. Grimmia Pennsyldanica.

Schwagr. - Sulliv. Icon. Musc. p. 68, t. 43.

HAB. ad rupes madidas in umbrosis montanis. 
211. Grimmia calyptrata.

Hook. - Sulliv. Icon. Musc. p. 69, t. 44.

$\mathrm{HAB}_{\mathrm{B}}$ in montosis Neo-Mexicanis (Wright legit).

\section{Grimina leucophan.}

Grev. - Bryol. Europ. Grimm. Monogr. p. 23, t. 20.

$\mathrm{HAB}_{\mathrm{A}}$, in rupibus Georgiæ et Ohionis.

\section{Grimisa ovata.}

Web. \& Molr. - Bryol. Europ. Grimm. Monogr.p. 21, t. 17.

$\mathrm{H}_{\mathrm{AB}}$. ad rupes in summo cacumine montis Mount-Marcy dicti, Reipublicæ Noræboracensis, alt. circa 6000 ped.

\section{Grinmita ovata.}

Var. AfFinis. - Bryol. Europ. Grimm. Monogr. p. 21, t. 18.

НАВ, in alpinis Montium-Rupestrium (Hall legit).

\section{Grimmia montana.}

Bryol. Europ. Grimm. Monogr. p. 26, t. 14.

$\mathrm{Var}$. foliis brevius pilifero-acuminatis, operculo peristomiique dentibus brevioribus, etc.

HАв. in rupibus schistosis montis Diablo Californiæ (Bolander); etiam in alpibus Sierra Nevada montium (Brewer).

216. Grimmia Donniana.

Smith. - Bryol. Europ. Grimm. Monogr. p. 20, t. 13.

НАв. in Montibus Albis Novæ Anglize (Oakes). 
217. RaCONITRIUM ACICULARE.

Brid. - Bryol. Europ. Racom. Monogr. p. 6, t. 1. $\mathrm{H}_{\mathrm{AB}}$. ad saxa, in rivulis per montes.

218. Racomitrium Sudeticum.

Brid. - Bryol. Europ. Racom. Monogr. p. 7, t. 1. НАв. in montibus editis Alleghaniensibus.

219. RACOMITRIUM MICROCARPON.

Brid. - Bryol. Europ. Racom. Monogr. p. 10, t. 5. НАв. in locis similibus cum priore.

2\%0. RaCOMItRIUM Fasciculare.

Brid. - Bryol. Europ. Racom. Monogr. p. 8, t. 4. НАв. ad rupes graniticas montosas.

221. Racomitrium CANESCENS.

Brid. - Bryol. Europ. Racom. Monogr. p. 12,t. 7 et 8. НАв. in alpinis Novæ Angliæ (Oakes).

222. Racomitrium canescens, var.

Foliis pellucide acuminatis angustius areolatis vix papillosis.

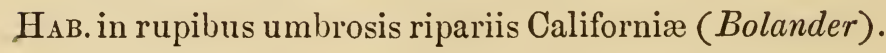

223. Raconitrium Lanuginosum.

Brid. - Bryol. Europ. Racom. Monogr. p. 11, t. 6. $\mathrm{H}_{\Delta \mathrm{B}}$. in montibus editioribus, ad saxa granitica. 


\section{Hedwigia ciliata.}

Ehrh. - Bryol. Europ. Hedw. Monogr. p. 5, t. 1 et 2. $\mathrm{H}_{\mathrm{A}}$. ad rupes graniticas erraticas ubique.

\section{Hedwigia ciliata, var.}

НАв. in rupibus irriguis Georgiæ superioris.

\section{Braunia Californica.}

Lesqx. in Trans. Amer. Phil. Soc. 13, p. 8.

Hedwigia pilifera, Mitten in Proceed. Linn. Soc. (1864), p. 45 .

НАB. in rupibus metamorphicis Californiæ montanæ, præcipue montis Diablo, ad 3000 ped. ascendens, perfrequens et variabilis (cl. Bolander legit).

227. Tetraplodon australis.

Sulliv. \& Lesqx. - Sulliv. Icon. Musc. p. 97, t. 58.

$\mathrm{H}_{\mathrm{AB}}$. ad mulorum stercorem in paludosis meridionalibus.

228. Tetraplodon angustatus.

Bryol. Europ. Tetrapl. Monogr. p. 4, t. 1.

НАв. in Montibus Albis Novæ Angliæ (Oakes); ad Lacus Superiorem (legit Loring).

\section{Tetraplodon MNioides.}

Hedw. - Bryol. Europ. Tetrapl. Monogr. p. 5, t. 2.

$\mathrm{H}_{\mathrm{AB}}$. in corporibus animalium putridis et stercore, per juga Montium Alborum Novæ Angliæ (James). 
230. Splachnum ampullaceum.

Linn. - Bryol. Europ. Splach. Monogr. p. 10, t. 6.

НАв. in turfosis montanis septentrionalibus.

231. Aphanoregma serratum.

(Hook \& Wils.) - Sulliv. Icon. Musc. p. 95, t. 57.

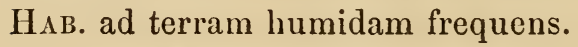

232. Physcomitrium tetragonun.

Bryol. Europ. Physc. Monogr. p. 9, t. 1.

Нав. in aridis orientalibus versus Montes-Rupestres (Hall legit).

233. Physcomitrium immersum.

Sulliv. Icon. Musc. p. 93, t. 56.

Нав. ad ripas fluviorum Pennsylvaniæ occidentalis.

234. Physconitrium pyriforme.

Bryol. Europ. Physc. Monogr. p. 11, t. 2.

$\mathrm{H}_{\mathrm{AB}}$. in agris argillosis ubique.

235. Entosthodon Drummondi.

Sulliv. Icon. Musc. p. 91, t. 55.

. НАв. in solo argilloso Alabamæ inferioris.

236. Entosthodon Bolanderi.

Lesqx. in Trans. Amer. Phil. Soc. 13, p. 10.

$\mathrm{H}_{\mathrm{AB}}$. ad terram argillosam prope San Francisco Californiæ (Bolander legit). 


\section{Entosthodon Templetonil.}

Schwagr. - Bryol. Europ. Entosth. Monogr. p. 3, t. 1.

$\mathrm{HAB}_{\mathrm{A}}$, in argillosis aridis Californiæ montanæ (Bolander).

238. Funaria Californica (n. $s p$.).

Pusilla; foliis erectis oblongis breviter acutis integris fere continuo-costatis; capsula erecta subpyriformioblonga æquali vel lenissime asymetrica, operculo depresso convexo-conico; pedicello sicco tota longitudine sinistrorsum torto; calyptra et peristomio generis.

$\mathrm{H}_{\mathrm{AB}}$ ad rupes et terram Californiæ montosæ (Bolander).

239. Funaria serrata.

Beauv. - Sulliv. Icon. Musc. p. 89, t. 54.

$\mathrm{HAB}_{\mathrm{A}}$ ad rivulorum margines, in arenosis Alabamæ superioris.

240. Funaria flavicans.

Michx. - Sulliv. Icon. Musc. p. 87, t. 53.

$\mathrm{H}_{\mathrm{AB}}$. in argillosis sylvaticis.

241. Funaria hygrometrica.

Hedw. - Bryol. Europ. Funar. Monogr. p. 8, t. 3.

$\mathrm{H}_{A B}$. ad terram murosque vulgatissima. 
242. Funaria hygronetrica.

Var. Calvescens, Bryol. Europ. l. c.

НАB. in locis herbidis humidis.

243. Amblyodon dealbatus.

Beauv. - Bryol. Europ. Amblyod. Monogr. p. 5, t. 1.

HАв. ad terram umbrosam in turfosis prope Milwaukee Reipublicæ Wisconsin (cl. I. A. Lapham comm.).

\section{Meesia Longiseta.}

Hedw. - Bryol. Europ. Mees. Monogr. p. 6, t. 2. НАв. in turfosis Ohionis superioris.

\section{Meesia Longiseta, var.?}

HAв. in montibus Noræ Angliæ (beat. Oakes legit).

\section{MeEsta tRISTICHA.}

Funk. - Bryol. Europ. Mees. Monogr. p. 8, t. 4. $\mathrm{HAB}_{\mathrm{B}}$ in turfosis profundioribus septentrionalibus.

\section{Meesia uliginosa.}

Hedw. - Bryol. Europ. Mees. Monogr. p. 5, t. 1.

$\mathrm{HAB}_{\mathrm{B}}$ in locis similibus cum præcedente.

\section{Meesia uliginosa.}

Var. alpina, Bryol. Europ. l. c.

$\mathrm{H}_{\mathrm{AB}}$. in turfosis montanis et septentrionalibus. 
249. Catoscopium nigritum.

Brid. - Bryol. Europ. Catosc. Monogr. p. 4, t. 1.

HAB. ad terram in Canada septentrionali.

250. Bartramia Calcarea.

Bryol. Europ. Bartram. Monogr. p. 19, t. 10.

Var.? foliis perigonialibus subobtusis costaque minus distincta. - Forsan ad B. fontanam pertinet.

HAB. in alpinis Noræ Angliæ (Oakes); in montibus editioribus septentrionalibus.

251. Bartrania Fontana.

Brid. - Bryol. Europ. Bartiam. Monogr. p. 18, t. 9.

HAB. ad fontes marginesque rivulorum in montosis.

\section{Bartramia fontana, var.}

$\mathrm{H}_{\mathrm{AB}}$. ad rupes irriguas in montibus Carolinarum.

\section{Bartramia Muhlenbergir.}

Schwagr. Suppl. I. 2, p. 58, t. 61. (A Bartramia Marchica vix distincta.)

HAB. ad fontes in sabulosis Ohionis.

254. Bartramia RADiCALIS.

Beauv. - Sulliv. Icon. Musc. p. 85, t. 52.

HAB. ad rupes arenosas madidas.

255. Bartramia ithyPhyLla.

Brid. - Bryol. Europ. Bartram. Monogr. p. 11, t. 2.

HAB. ad rupes per montes Alleghanienses. 
256. Bartramia stricta.

Brid. - Bryol. Europ. Bartram. Monogr. p. 10, t. 1.

A forma normali tantum pedicello superne cylindrico (nec obtuse tetragono) differt.

$\mathrm{H}_{\mathrm{AB}}$. in rupibus humidis prope San Francisco Californiæ, rara (Bolander legit).

257. Bartramia pomiformis.

Hedw. - Bryol. Europ. Bartram. Monogr. p. 13, t. 4. $\mathrm{H}_{\mathrm{AB}}$. in umbrosis argillosis sylvarum.

\section{Bartrania Eederi.}

Swartz. - Bryol. Europ. Bartram. Monogr. p. 12, t. 3. $\mathrm{H}_{\mathrm{AB}}$ ad rupes in montibus Noveboracensis.

259. Bartramia Menziesir.

Turn. - Hook. Musc. Exot. t. 67.

Glyphocarpa Baueri, Hampe in Linnaa (1859), p. 457. $\mathrm{H}_{\mathrm{AB}}$. in rupibus umbrosis Californiæ Inferioris, perfrequens (Bolander).

\section{Bartramia Menziesit, var.}

Foliis humiditate magis apertis basi latioribus brevius acuminatis; capsula longiore ovato-subcylindrica, ore latiori nudo vel tantum membrana integra circumdato.

$\mathrm{H}_{\mathrm{AB}}$. in rupibus aridis Californiæ (Bolander). 


\section{Conostomum boreale.}

Swartz. - Bartramia conostoma, Bryol. Europ. Bartram. Monogr. p. 16, t. 7.

$\mathrm{H}_{\mathrm{AB}}$. in alpinis Montium Alborum Noræ Angliæ (legit Oakes).

\section{Bryum CernuUm.}

Hedw. - Bryol. Europ. Bry. Monogr. p. 14, t. 1.

$\mathrm{HAB}_{\mathrm{B}}$ ad terram in Ohione inferiore.

263. Bryum uliginosum.

Brid. - Bryol. Europ. Bry. Monogr. p. 18, t. 4.

$\mathrm{HAB}_{\mathrm{B}}$ in ligno putrido, ad fontes frigidas Ohionis superioris, et in locis similibus Minnesotæ.

264. Bryum aCuminatum.

Hoppe \& Hornsch. - Bryol. Europ. Bry. Monogr. p. 21, t. 6.

НАв. in fissuris rupium montium Adirondack, rarum.

265. Bryun cucullatum.

Schwagr. - Bryol. Europ. Bry. Monogr. p. $27, t .8$.

Haв. in alpibus Novæ Angliæ, rarum (Oakes).

266. Bryum elongatum.

Dicks. - Bryol. Europ. Bry. Monogr. p. 32, t. 10 et 11.

$\mathrm{H}_{A B}$. ad terram humosam in montibus editioribus. 
267. Bryun nutans.

Schreb. - Bryol. Europ. Bry. Monogr. p. 34, t. 12.

НАв. ad rupes terramque haud infrequens.

268. BRyum nutans.

Var. BICOLor, Bryol. Europ. l. c.

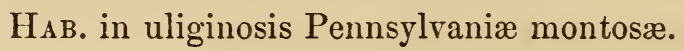

269. Bryum CRUdum.

Schreb. - Bryol. Europ. Bry. Monogr. p. 67, t. 13.

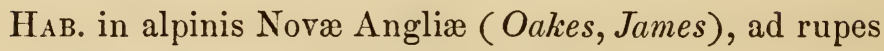
Lacus Superioris (comm. Agassiz).

270. Bryum Ludwigir.

Spreng. - Bryol. Europ. Bry. Monogr. p. 38, t. 14.

$\mathrm{H}_{\mathrm{AB}}$. in alpinis Montium-Rupestrium (Hall parce legit et comm.).

\section{Bryum annotinum.}

Hedw. - Bryol. Europ. Bry. Monogr. p. 40, t. 15. (Varietas robustior.)

$\mathrm{H}_{A B}$. in Novæ Angliæ montosis.

\section{BRyum TOZeri.}

Grev. - Bryol. Europ. Bry. Monogr. p. 41, t. 16.

$\mathrm{H}_{\mathrm{AB}}$. in argillosis ad margines viarum et fossarum Californiæ inferioris, frequens (Bolander comm.). 
273. Bryum Lescurianum.

Sulliv. Icon. Musc. p. 81, t. 50.

$\mathrm{H}_{\mathrm{AB}}$. ad terram sub Rhododendrontis maximi umbra, in collinis Ohionis et Pennsylvaniæ, rarius.

\section{Bryum WaHLENBERgiI.}

Schwagr. - Bryol. Europ. Bry. Monogr. p. 44, t. 17.

$\mathrm{HAB}_{\mathrm{A}}$. in sabulosis scaturiginosis, haud rarum.

\section{Bryum WAHLENBERgit, var.}

НАв. ad rupes irriguas Californiæ (Bolander).

276. BRYUM PYRIFORME.

Hedw. - Bryol. Europ. Bry. Monogr. p. 45, t. 18.

НАв. in umbrosis ad terram, frequens.

\section{BRYUM INTERMEDIUN.}

Brid. - Bryol. Europ. Bry. Monogr. p. 47, t. 19.

$\mathrm{H}_{\mathrm{AB}}$. in fissuris rupium, frequens.

\section{Bryun Bimum.}

Schreb. - Bryol. Europ. Bry. Monogr. p. 50, t. 21.

$\mathrm{H}_{\mathrm{AB}}$. ad arborum radices, in paludibus sylvaticis vulgare.

\section{Bryum PaLlescens.}

Schwagr. - Bryol. Europ. Bry. Monogr. p. 51, t. 22. $\mathrm{H}_{\mathrm{AB}}$. ad rupes arenaceas humidas Ohionis inferioris, per rarum. 
280. Bryum torquescens.

Bryol. Europ. Bry. Monogrr. p. 49, t. 20.

HAB. in terris Neo-Mexicanis (legit Wright).

281. BRyum PSEUdo-triquetrum.

Schwagr. - Bryol. Europ. Bry. Monogr. p. 54, t. 24.

$\mathrm{H}_{\mathrm{AB}}$. in rupibus irriguis Ohionis inferioris.

282. BRYUM ROSEUMr.

Schreb. - Bryol. Europ. Bry. Monogr. p. 56, t. 25.

$\mathrm{H}_{\mathrm{AB}}$. ad terram umbrosam truncosque putridos, frequens, sed rarius fructificans.

283. Bryum occidentale.

Sulliv. in Bot. Pacif. R. R. Survey (Whipple), p. 188, t. 7.

$\mathrm{H}_{\mathrm{AB}}$. ad terram et rupes Californix, perfrequens et variabile (Bolander comm.).

284. Bryum occidentale, var.

B. obconicum, Musc. Exsic. Amer. No. 187 ${ }^{\text {b }}$ ed. 1.

$\mathrm{H}_{\mathrm{AB}}$. in truncis emortuis et deustis Californiæ (Bigelow et Bolander comm.).

285. Bryum Capillare.

Hedw. - Bryol. Europ. Bry. Monogr. p. 60, t. 28.

$\mathrm{H}_{\triangle \mathrm{B}}$. ad viarum margines præruptas, in montosis umbrosis Pennsylvaniæ. 
286. Bryum DuvaliI.

Voit. - Bryol. Europ. Bry. Monogr. p. 64, t. 31.

НАв. in montibus editioribus Noræ Angliæ (Oakes).

287. Bryun turbinatum.

Hedw. - Bryol. Europ. Bry. Monogr. p. 65, t. 32.

$\mathrm{H}_{\mathrm{AB}}$ ad rupes irroratas Niagara cataractæ; etiam in scaturiginosis Mimnesotæ.

288. BRyun CAEsPITICIUM.

Linn. - Bryol. Europ. Bry. Monogr. p. 70, t. 34.

$\mathrm{H}_{\mathrm{AB}}$. in locis arenosis siccioribus.

289. BRyUM CASPITICIUn, var.

$\mathrm{H}_{\mathrm{AB}}$. in umbrosis humidis ad terram murosque.

290. Bryum ARGENTEUM.

Linn. - Bryol. Europ. Bry. Monogr. p. 78, t. 41.

$\mathrm{HAB}_{\mathrm{B}}$ in terris siccis arenosis vulgare.

291. Bryum atropurpureum.

Web. et Mohr. - Bryol. Europ. Bry. Monogr. p. 73, t. 37.

НАв. ad margines viarum, in solo arenoso Alabamæ montosæ. 
292. Bryum Californicum.

Sulliv. in Bot. Pacif. R. R. Survey (Whipple), p.188, t. 6.

$\mathrm{H}_{\mathrm{AB}}$. ad terram in dumetis et herbosis Californiæ (Bolander comm.).

\section{BRyum alpinum.}

Linn. - Bryol. Europ. Bry. Monogr. p. 76, t. 39.

$\mathrm{H}_{\mathrm{AB}}$. in alpibus Novæ Angliæ (Oakes).

\section{Mnium punctatum.}

Hedw. - Bryol. Europ. Mn. Monogr. p. 19, t. 2.

$\mathrm{HAB}_{\mathrm{A}}$ ad terram truncosque putridos, in irriguis montosis umbrosis.

\section{Mnium Cinclidioides.}

Hubener. - Bryol. Europ. Mn. Monogr. p. 34, t. 14.

$\mathrm{H}_{\mathrm{AB}}$. in turfosis montis Novæ Angliæ(Eaton, Ingraham).

296. MNium hoRnum.

Hedw. - Bryol. Europ. Mn. Monogr. p. 22, t. 4.

$\mathrm{H}_{\mathrm{AB}}$. in alpinis Novæ Angliæ (Oakes).

\section{Mnium serratum.}

Brid. - Bryol. Europ. Mn. Monogr. p. 24, t. 5.

$\mathrm{H}_{\mathrm{AB}}$. in terra sabulosa, ad marginem rivulorum sylvestrium, rarius. 
298. Mnium Lycopodioides.

Hook. - Bryol. Europ. Mn. Monogr. Suppl. I. p. 3, t. Suppl. 2.

$\mathrm{H}_{\mathrm{AB}}$. in rupibus irriguis et umbrosis montanis.

\section{Mnium Drummond.}

Bruch \& Schimp. - Sulliv. Icon. Musc. p. 83, t. 51.

$\mathrm{H}_{\mathrm{AB}}$. in montibus Novæ Angliæ (Oakes legit).

300. MNium cuspidatum.

Hedw. - Bryol. Europ. Mn. Monogr. p. 29, t. 8.

$\mathrm{H}_{\mathrm{AB}}$ ad arborum radices in sylvis.

\section{Mnium affine.}

Bland. - Bryol. Europ. Mn. Monogr. p. 30, t. 9.

$\mathrm{H}_{\mathrm{AB}}$. in iisdem locis cum priore.

302. Mnium AfFine.

Var. Elatum, Bryol. Europ. Mn. Monogr. p. 31, t. 10.

$\mathrm{HAB}_{\mathrm{A}}$. in umbrosis humidis montanis.

303. Mnium affine.

Var. Rugicum, Bryol. Europ. Mn. Monogr. p. 31, t. 11.

HAB. cum præcedente.

304. Mnium spinulosum.

Bryol. Europ. Mn. Monogr. Suppl. I. p. 4, t. 4.

НАв. in montibus Nогæ Angliæ (Oakes). 


\section{Mnium stellate.}

Hedw. - Bryol. Europ. Mn. Monogr. p. 33, t. 13.

$\mathrm{H}_{\mathrm{AB}}$ ad terram humosam in abruptis umbrosis, rarum.

306. Mnium Menziesir.

Hook. in Bot. Miscell. 1, p. 36, t. 19.

$\mathrm{H}_{\mathrm{AB}}$. in rupibus umbrosis ad ripas rivulorum Californiæ (Bolander pulcherrime legit et comm.).

307. Aulacominion turgidum.

Schwagr. - Bryol. Europ. Aulacom. Monogr. p. 8, t. 2. НАв. in turfosis Noræ Angliæ montosæ.

308. Aulacominion palustre.

Schwagr. - Bryol. Europ. Aulacom. Monogr. p. 9, t. 3. $\mathrm{H}_{\mathrm{AB}}$, in turfosis vulgare.

309. Aula Connion androgynum.

Schwagr.-Bryol. Europ. Aulacom. Monogr. p. 11, t.4. $\mathrm{H}_{\mathrm{AB}}$. in truncis emortuis, per Californiam sat frequens (legit Bigelow, Bolander).

310. Aulacomnion heterostichum.

Bryol. Europ. Aulacom. Monogr. p. 7, t. 1.

$\mathrm{H}_{\mathrm{AB}}$. ad terram humidam, in declivibus sylvaticis frequens.

311. Timmia megapolitana.

Hedw. - Bryol. Europ. Tim. Monogr. p. 6, t. 1.

$\mathrm{H}_{\mathrm{AB}}$. in præruptis sylvaticis ad terram rupesque. 
312. Timmia Austriaca.

Hedw. - Bryol. Europ. Tim. Monogr. p. 7, t. 2.

НАв. in alpinis Montium-Rupestrium (Hall legit).

313. Atrichun undulatum.

Beauv. - Bryol. Europ. Atrich. Monogr.p. 8, t. 1 et 2.

$\mathrm{H}_{\mathrm{AB}}$ in sylvis argillaceo-arenosis vulgare.

314. Atrichum angustatun.

Hook. - Bryol. Europ. Atrich. Monogr. p. 9, t. 3.

$\mathrm{H}_{\mathrm{AB}}$. in locis similibus cum præcedente.

315. Atrichum CRISPUM.

James. - Sulliv. Icon. Musc.p. 73, t. 46.

$\mathrm{H}_{\mathrm{AB}}$ ad margines rivulorum Novæ Cæsaræ (James).

316. Pogonatum Brevicaule.

Brid. - Sulliv. Icon. Musc. p. 75, t. 47.

$\mathrm{HAB}_{\mathrm{AB}}$ in terra argillosa.

317. Pogonatum BRachyphyllum.

Beauv. - Sulliv. Icon. Musc. p. 77, t. 48.

$\mathrm{H}_{\mathrm{AB}}$. in argillaceo-arenosis Georgiæ atque Carolinæ utriusque.

318. Pogonatum capillare.

Michx., Brid. - Sulliv. Icon. Musc. p. 79, t. 49.

Нав. in Montibus Albis Novæ Angliæ (Oakes). 
319. Pogonatum urnigerum.

Brid. - Bryol. Europ. Pogonat. Monogr. p. 7, t. 9.

$\mathrm{H}_{\mathrm{AB}}$. per montes Alleghanienses.

\section{Pogonatum alpinum.}

Brid. - Bryol. Europ. Pogonat. Monogr. p. 9, t. 10.

$\mathrm{H}_{\mathrm{AB}}$. in montibus editioribus; etiam in sylvis Lacus Superioris.

321. Pogonatum alpinum, var.

НAв. in Montibus-Rupestribus (Hall legit).

322. Polytrichadelphus Lyallit.

Mitten in Jour. Linn. Söc. (1864), p. 49.

$\mathrm{HAB}_{\mathrm{AB}}$ in sylvis Montium-Rupestrium (Hall); in montibus altioribus Californiæ (Bolander).

323. Polytrichum Formosum.

Hedw. - Bryol. Europ. Polytr. Monogr. p. 9, t. 12.

$\mathrm{H}_{\mathrm{AB}}$. in sylvis ad terram, haud rarum.

324. Polytrichum gRacile.

Menzies. - Bryol. Europ. Polytr. Monogr.p. 10, t. 13. $\mathrm{HAB}_{\mathrm{A}}$ in turfosis calidioribus.

325. Polytrichum piliferum.

Schreb. - Bryol. Europ. Polytr. Monogr. p. 11, t. 14. $\mathrm{H}_{\mathrm{AB}}$. in arenosis siccis montosis. 
326. Polytrichum piliferum.

Schreb. - Var. LæVIPILUM.

P. lævipilum, Hampe in Linnæa (1859), p. 459.

$\mathrm{H}_{\mathrm{AB}}$. per regiones Montium Rupestrium (Hall), et in montibus Californiæ (Bolander).

327. Polytrichiom juniperinum.

Hedw.-Bryol. Europ. Polytr. Monogr. p. 12, t. 15 et 16. $\mathrm{H}_{\mathrm{AB}}$, in sylvis montosis.

328. Polytrichum JUNiPERINum.

Var. Alpestre, Bryol. Europ. l. c.

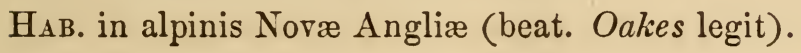

329. Polytrichum cominue.

Linn. - Bryol. Europ. Polytr. Monogr. p. 13, t. 17.

$\mathrm{H}_{\mathrm{AB}}$. in arenosis turfosis præprimis montosis.

330. Polytrichum cominune, var.

НАв. in montibus Noræ Angliæ (beat. Oakes legit).

331. Buxbaumia aphylla.

Haller. - Bryol. Europ. Bux. Monogr. p. 5, t. 1.

НАв. in locis graniticis Novæ Angliæ (beat. Oakes comm.).

332. Diphyscium foliosum.

Web. \& Mohr. - Bryol. Europ. Diphysc. Monogr. p. $3, t .2$.

HAB. in sylvaticis umbrosis argillosis, ad margines viarum. 
333. Fontinalis antipyretica.

Linn. - Bryol. Europ. Fontin. Monogr. p. 5, t. 2.

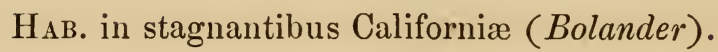

\section{Fontinalis antipyretica, var.}

F. Neo-Mexicana, Sulliv. \& Lesqx. Musc. Exsicc. ed. 1, No. $224^{\mathrm{b}}$.

НАB. in rivulis montosis Neo-Mexicanis (Wright); in Montibus-Rupestribus pulcherrime fructificans (Hall).

335. Fontinalis antipyretica.

Var. gigantea, Sulliv. Icon. Musc. p. 106, t. 66.

НАв. in rivulis montosis Novæ Angliæ et Novæboracensis.

\section{Fontinalis Nove Angliz.}

Sulliv. Icon. Musc. p. 105, t. 65.

$\mathrm{H}_{\mathrm{AB}}$. in rivulis Novanglis sterilis (comm. Oakes, Olney, Eaton); specimina fructifera James legit.

\section{Fontinalis biformis.}

Sulliv. Icon. Musc. p. 99, t. 59.

(Forma vernalis).

НАв. ad lapides in rivulis sylvaticis prope Columbum in Ohione, raro fructificans.

338. Fontinalis biformis.

Sulliv. Icon. Musc. p. 99, t. 60.

(Forma æstivalis).

НАB. in iisdem locis ac præcedens. 
339. Fontinalis disticha.

Hook. \& Wils. - Sulliv. Icon. Musc. p. 103, t. 63, 64. НАв. in rivulis prope Mobile, Alabamæ; etiam in Kentucky australi.

\section{Fontinalis Lescurit.}

Sulliv. Icon. Musc. p. 101, t. 61.

НАB. in rivulis montanis a Nova Anglia usque ad Alabamam, haud rara.

341. Fontinalis Lescurit, var. $\gamma$.

Sulliv. Icon. Musc. p. 101.

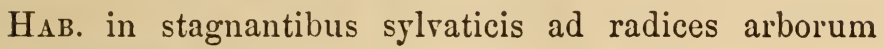
(Austin legit).

\section{Fontinalis Dalecarlica.}

Bryol. Europ. Fontin. Monogr. Suppl. I. p. 7, t. 1. $\mathrm{H}_{A B}$. in rivulis montanis vulgaris.

343. Dichelyma falcatum. Myrin.-Bryol. Europ. Dichel. Monogr. p. 6, t. 1. $\mathrm{H}_{\mathrm{AB}}$. in rivulis Novanglis montosis (James legit).

\section{Dichelyan Swartzit.}

Lind. - Schimp. Syn. Musc. Europ. p. 461.

$\mathrm{H}_{\mathrm{AB}}$. in rivulis pinetorum Neo-Cæsariensium (Austin); etiam in montibus Californice (Brewer); semper sterile. 
345. Dichelyma capillaceum.

Dill. - Bryol. Europ. Dichel. Monogr. Suppl. I. p. 1, t. Suppl. 1.

$\mathrm{H}_{\mathrm{AB}}$, in rivulis sylvaticis montanis.

346. Dichelyma pallescens.

Bryol. Europ. Dichel. Monogr. Suppl. I. p. 2, t. 3.

НАв. in stagnantibus umbrosis Noræ Angliæ (Ingraham legit).

347. Cryphea glomerata.

Schimp. - Sulliv. Icon. Musc. p. 107, t. 67.

HАB. ad arborum corticem in Carolinis utrisque.

348. Pterigynandrum filiforme.

Hedw. - Bryol. Europ. Plerigyn. Monogr. p. 3, t. 1.

$\mathrm{H}_{\mathrm{AB}}$. ad radices arborum in montibus Novanglis et Novæboracensibus.

349. Pterogonium gracile.

Swartz. - Bryol. Europ. Plerog. Monogr. p. 4, t. 1.

$\mathrm{H}_{\mathrm{AB}}$ ad rupes Californiæ, perfrequens (Bolander).

350. LEUCODON JULACEUS.

(Hedw.) Schwagr. - Sulliv. Icon. Musc. p. 110, t. 69. НАв, ad arbores planitierum, montosa fugiens.

351. LeUCOdON BRACHYPUS.

Brid. - Sulliv. Icon. Musc. p. 111, t. 70.

$\mathrm{HAB}_{\mathrm{B}}$ ad rupes et arbores in sylvis montanis. 
352. LEPTODON TRICHOMITRION.

Mohr. - Sulliv. Icon. Musc. p. 112, t. 71.

$\mathrm{H}_{\mathrm{AB}}$. ad arbores in umbrosis.

353. Leptodon trichonitrion.

Tar. Immersus.

НАB. ad arbores in Rebuspublicis meridionalibus.

\section{Leptodon Ohioense.}

Sulliv. Icon. IIusc. p. 114, t. 72.

$\mathrm{H}_{\mathrm{AB}}$. ad arbores in sylvis profundis.

355. ANtitrichia CURTipendula.

Brid. - Bryol. Europ. Antitr. Monogr. p. 2, t. 1.

$\mathrm{H}_{\mathrm{AB}}$. in summo cacumine Black montis Carolinæ Septentrionalis; etiam in sylvis circa Lacum Superiorem.

356. Antitrichia CURTipendula.

Var. gigantea.

$\mathrm{H}_{\mathrm{AB}}$. in sylvis Californiæ montosæ ad rupes et arbores (Bolander).

357. Antitrichia Californica.

Sulliv. - Trans. Amer. Phil. Soc. p. 11.

$\mathrm{H}_{A B}$. in sylvis Californiæ inferioris frequens, arborea, (Bolander fertilem legit). 
358. Anomodon apiculatus.

Bryol. Europ. Mem. in Anom. Monogr.p. 3 et 6 . НАВ. ad rupes in sylvis montanis.

359. ANomodon viticulosus.

Hook. \& Tayl. - Bryol. Europ. Anom. Monogr. p. 5, t. 3.

$\mathrm{H}_{\mathrm{AB}}$ ad rupes humidas Niagarce cataractæ.

360. ANOMODON OBTUSIFOLIUS.

Bryol. Europ. - Sulliv. Icon. Musc. p. 119, t. 74.

НАв. in sylvis humidis ad arbores.

361. ANomodon attendatus.

Hartm. - Bryol. Europ. Anom. Monogr. p. 4, t. 1.

HAB. ad arborum radices terramque humosam, præprimis secus rivulos sylvarum.

362. Anomodon? Toccoz.

Sulliv. \& Lesqx. - Sulliv. Icon. Musc. p. 121, t. 76.

НАв. ad rupes prope Toccoa Falls Georgiæ superioris, sterilis.

363. ANOMOdON? TRISTIS.

(Cesati) Hook. \& Tayl. - Hypnum triste, C. Mull. Synops. Musc. 2, p. 478. - Leskea fragilis, Hook. \&. Wils. in Drumm. Musc. Amer. coll. 2, No. 101.

НАв. ad arbores in sylvis humidis, præprimis secus rivulos, semper sterilis. 
364. Leskea POLYCARPA.

Hedw. - Bryol. Europ. Lesk. Monogr. p. 2, t. 1.

HAB. ad arbores inundatas.

365. LESKEA OBSCURA.

Hedw. - Sulliv. Icon. Musc. p. 123, t. 77.

$\mathrm{H}_{\mathrm{AB}}$. in iisdem locis cum præcedente, vulgaris.

366. Leskea rostrata.

Hedw. - Bryol. Europ. Lesk. Monogr. p. 4, t. 4.

$\mathrm{H}_{\mathrm{AB}}$. ad arborum radices, dense cæespitosa.

367. Leskea dexticulata.

Sulliv. Icon. Musc. p. 125, t. 78.

$\mathrm{H}_{\mathrm{AB}}$. ad arbores in montibus meridionalibus, sæpius sterilis.

368. Clasilatodon parvulus.

(Hampe) Hook. \& Wils. - Sulliv. Icon. Musc. p. 126, t. 79 .

НАв. ad arbores in Rebuspublicis meridionalibus.

369. Clasmatodon parvulus.

Hampe. - Var. RUPESTRIS.

$\mathrm{H}_{\mathrm{AB}}$. in rupibus inundatis fluminis Tennessee.

370. Thelia hirtella.

(Hedw.) - Sulliv. Icon. Musc. p. 128, t. 80.

$\mathrm{HAB}_{\mathrm{A}}$ ad arborum truncos radicesque, vulgaris. 


\section{Thelia asprella.}

(Schimp.) - Sulliv. Icon. Musc. p. 129, t. 81.

НАB. cum præcedente.

\section{Thelia Lescurit.}

Sulliv. Icon. Musc. p. 130, t. 82.

$\mathrm{HAB}_{\mathrm{AB}}$ ad terram in sabulosis siccioribus collinis utriusque Carolinæ et Georgiæ.

\section{Myurella Careyana.}

Sulliv. Icon. Musc. p. 131, t. 83.

Hав. ad rupes irriguas in montibus Noræ Angliæ Pennsylvaniæ et Carolinarum.

\section{Fabronia Wrightil.}

Sulliv. Icon. Musc. p. 133, t. 84 .

НАв. in agro Texano (Wright legit).

\section{Fabronia pusilla.}

Raddi._Bryol. Europ. Fabron. Monogr. p. 3, t. 1.

$\mathrm{H}_{\triangle B}$. in cortice arborum Californiæ Inferioris (Bolander legit).

376. Fabronia Ravenelit.

Sulliv. Icon. Musc. p. 135, t. 85.

HAB. ad rupes siccas Carolinæ Inferioris (Ravenel).

377. Fabronia Ravenelit, var.

HAB. ad truncos putridos Carolinæ Inferioris (Ravenel). 
378. Fabronia grinostoma.

Sulliv. \& Lesqx. - Sulliv. Icon. Musc. p. 136, t. 86.

Haв. prope Santa Fé Neo-Mexicanorum (Wriglıl).

379. Anacamptodon splachioides.

Brid. - Bryol. Europ. Anacamp. Monogr. p. 3, t. 1.

$\mathrm{H}_{\mathrm{AB}}$. in arborum nodis apertis putridine excaratis, rarus.

380. Pylaisana intricata.

(Hedw.) W. P. Schimp. - Sulliv. Icon. Musc. p. 139, t. 88.

$\mathrm{H}_{\mathrm{AB}}$. ad arborum corticem in sylvis, vulgaris.

381. Pylaisea velutina.

Bryol. Europ. Mem. - Sulliv. Icon. Musc. p. 140, t. 89. $\mathrm{H}_{\mathrm{AB}}$. in sylvis editioribus, arborea.

382. Pylaisea subdenticulata.

Sulliv. Icon. Musc. p. 137, t. 87.

$\mathrm{HAB}_{\mathrm{A}}$. in sylvis ad arborum corticem, rara.

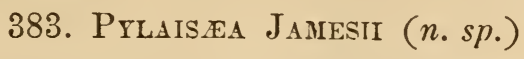

P. subdenticulatæ simillima; recedit tamen colore sordide viridi, cæspitibus densioribus, ramis longioribus erectis, foliis undique imbricatis brevioribus, præsertim operculo duplo breviore, etc.

НАB. in terra ad radices arborum prope Chelseam Nove Angliæ (cl. T. P. James legit). 
384. Homalothecium subcapillatum.

(Hedw.) Bryol. Europ. - Sulliv. Icon. Musc. p. 141, t. 90.

$\mathrm{H}_{\mathrm{AB}}$, in cortice arborum, haud rarum.

385. Platygyrium repens.

Bryol. Europ. Platyg. Monogr.p. 4, t. 1.

Hab. ad truncos putridos sylvarum.

386. Cylindrothecium cladorrhizans.

(Hedw.) Bryol. Europ. - Sulliv. Icon. Musc. p. 143, t. 91 .

HAB. ad terram truncosque prostratos in sylvis, frequens.

387. Cylindrothecium Seductrix.

(Hedw.) Bryol. Europ. - Sulliv. Icon. Musc. p. 145, t. 92.

$\mathrm{H}_{\triangle B}$. ad truncos emortuos in sylvis humidis, frequens.

388. Cylindrothecium compressum.

(Hedw.) Bryol. Europ. - Sulliv. Icon. Musc. p. 147, t. 93.

HAB. ad arborum radices secus fluviorum ripas, rarum.

389. Cylindrothecium Sullivantir.

(C. Mull.) Bryol. Europ. - Sulliv. Icon. Musc.p. 149, t. 95 .

HАв. in Tennessee et Carolina Inferiore, ad saxa in sylvaticis. 
390. Cylindrotheciun Drumandi.

Bryol. Europ. - Sulliv. Icon. Musc. p. 148, t. 94.

HAB. ad saxa truncosque putridos, in sylvis Georgiæ et Carolinarum.

391. Cylindrothecium BRevisetum.

(Wils.) Bryol. Europ. - Sulliv. Icon. Musc. p. 150, t. 96 .

$\mathrm{H}_{A B}$. in truncis emortuis sylvarum.

392. Neckera pennata.

(Hedw.) Bryol. Europ. Neck. Monogr. p. 6, t. 1.

$\mathrm{H}_{\mathrm{AB}}$. in sylvis montosis, arborea et rupestris.

393. Neckera oligocarpa.

Bryol. Europ. Neck. Monogr. p. 7, t. 2.

Neckera chlorocaulis Musc. Exsicc. No. 268, ed. 1.

Hab. juxta Santa Fé Neo-Mexicanorum (legit Fendler).

394. Neckera DouglassiI.

Hook. in Bot. Misc. 1, p. 131, t. 35.

$\mathrm{HAB}_{\mathrm{A}}$ ad rupes umbrosas in California (Bolander).

395. Neckera Menziesir.

Hook. in Drum. Musc. Amer. No. 162.

HAB. ad rupes in umbrosis profundis Californiæ (pulcherrime fructificantem (Bolander legit et comm.). 
396. Neckera complanata.

Hedw. - Bryol. Europ. Necker. Monogr. p. 9, t. 5.

$\mathrm{H}_{\mathrm{AB}}$ ad rupes umbrosas Tennessee, sterilis.

\section{Omalia Janesit.}

Schimp. Syn. Musc. Europ. Annot.p. 473.

Hab. in montibus Novæ Angliæ ad rupes (James legit et comm.).

\section{Alsia Californica.}

(Hook. \& Arn.) - Sulliv. Musc. U. S. S. Pacif. Explor. Exped. (Wilkes), p. 25, t. 25.

$\mathrm{H}_{\mathrm{AB}}$. ad arborum corticem in California, haud rara (Bigelow, Bolander).

\section{Alsia longipes ( $n . s p$.).}

Dioica, pinnato-ramulosa, subfrondiformis, compressiuscule foliosa ; foliis oblongo-lanceolatis breviter acutatis superne grosse serratis minute ovali-areolatis, costa ultramedia apice dorsali denticulata; perichætialibus abrupte filiformi-attenuatis; capsula cylindracea in pedicellum subuncialem perichætio quadruplo longiorem defluente; peristomio 1-2-ciliolato; operculo calyptraque A. abietinæ.

НАв. in profundis convallibus ad rupes Californiæ (legit Bolander).

400. Alsia abietina.

(Hook.) - Sulliv. Icon. Musc.p. 115, t. $72^{\mathrm{b}}$.

$\mathrm{H}_{\mathrm{AB}}$ ad arbores in sylvis Californiæ frequens (Bolander comm.). 
401. HoOKeria ACUTIFOLIA ?

Hook.; Schwagr. Suppl. II. 2, p. 36, t. 163.

$\mathrm{H}_{\triangle B}$. ad rupes irriguas in montosis, sterilis, rarissima.

402. Climacium americanum.

Brid. - Sulliv. Icon. Musc. p. 151, t. 97.

$\mathrm{H}_{\Lambda \mathrm{B}}$ ad truncos putridos in sylvis humidis.

403. Hypnum tamariscinum.

Hedw.-Thuidium tamariscinum, Bryol. Europ. Thuid. Monogr.p. 7, t. 2 et 3.

$\mathrm{H}_{\Lambda \mathrm{B}}$ ad truncos terramque in sylvis, vulgatissimum.

404. Hypnum delicatulum.

Linn. - Thuidium delicatulum, Bryol. Europ. Thuid. Monogr. p. 8, t. 4.

$\mathrm{H}_{A B}$. ad margines sylvarum, terrestre, rarum.

\section{Hypnum CRispifolium.}

Hook. Musc. Exot. t. 31.

$\mathrm{H}_{\mathrm{AB}}$. ad terram umbrosam et rupes in sylvis Californix (Bolander comm.).

\section{Hypnum minutulum.}

Hedw. - Thuidium minutulum, Bryol. Europ. Thuid. Monogr. p. 5, t. 1.

НАв. in ligno putrido sylvarum. 


\section{Hypnum Whippleanum.}

Sulliv. in Bot. Pacif. R. R. Survey (Whipple), p. 190, t. 9 .

Pedicello constanter scabro, haud variabili ut in Sulliv.

Icon. Musc. p. 166, memorato.

HAB. ad terram, in convallibus montium prope sinus San Francisco, Californiæ (Bigelow).

\section{7 b. Hypnum Leuconeuron (n. $s p$.)}

Hypno Whippleano per affine, cæspite densiore, foliis confertioribus erectioribus, operculo breviore, pedicello lævi, etc. distinguendum.

$\mathrm{HAB}_{\mathrm{A}}$ ad truncos Quercus agrifolia in sylvis Californiæ inferioris (Bolander legit).

408. HYPNUM PYGMÆUM.

Bryol. Europ. Mem. - Sulliv. Icon. Musc. p. 153, t. 98. HAB. ad saxa, in depressis humidis Ohionis.

409. Hypnum SCITUM.

Beauv. - Sulliv. Icon. Musc. p. 155, t. 99.

$\mathrm{HAB}_{\mathrm{A}}$ ad radices arborum præsertim fagorum.

410. Hypnum gracile.

Bruch \& Schimp. - Sulliv. Icon. Musc. p. 156, t. 100. $\mathrm{H}_{\mathrm{AB}}$ ad truncos emortuos in sylvis humidis. 


\section{Hypnum gracile.}

Var. Lancastriense. Foliis brevioribus appressis apice serrulatis valde papillosis, perichætio virescente, operculo longiore, capsulaque graciliore. - An species propria?

$\mathrm{H}_{A B}$. ad arborum radices, in collibus siccis Ohionis.

\section{Hypnum GRacile.}

Var. RAVENeLII. Statura minore graciliore, foliis margine subplanis, areolatione densiore, pedicello breviore cygneo, peristomii ciliolis appendiculatis, operculo longiore. - Forsan species propria.

$\mathrm{H}_{\mathrm{AB}}$ ad muros lateritios Carolinæ Meridionalis.

\section{Hypudu abietindir.}

Linn. - Thuidium abietinum, Bryol. Europ. Thuid. Monogr. p. 9, t. 5.

НАв. in sylvis Lacus Superioris (cl. Agassiz comm.); etiam in insula Goat Island dicta juxta cataractam Niagarce.

\section{Hypnum Blandowir.}

Web. \& Mohr. - Thuidium Blandowii, Bryol. Europ. Thuid. Monogr. p. 10, t. 6.

$\mathrm{H}_{\mathrm{AB}}$. in turfosis Wisconsin (Lapham legit).

\section{Hypnum paludosum.}

Sulliv. Icon. Musc. p. 157, t. 101.

$\mathrm{H}_{\mathrm{AB}}$. in turfosis paludibusque sylvaticis Ohionis et Noræ Angliæ. 
416. Hypnum squarrosum.

Linn. - Hylocomium squarrosum, Bryol. Europ. Hylocom. Monogr. p. 9, t. 6 .

HAв. ad margines sylvarum, in humidis herbosis Pennsylvaniæ, sterile.

417. Hypnum triquetrum.

Linn. - Hylocomium triquetrum, Bryol. Europ. Hylocom. Monogr. p. 8, t. 5 .

$\mathrm{H}_{A B}$. in sylvis montosis abietinis.

418. Hypnum BRevirostre.

Elhr. - Hylocomium brevirostre, Bryol. Europ. Hylocom. Monogr. p. 10, t. 7.

$\mathrm{H}_{\mathrm{AB}}$. in summis jugis Alleghaniensium.

419. Hypnum splendens.

Hedw. - Hylocomium splendens, Bryol. Europ. Hylocom. Monogr. p. 5, t. 1 .

$\mathrm{H}_{\mathrm{AB}}$. in sylvis montosis, frequens.

\section{Hypnum umbratum.}

Elrrh. - Hylocomium umbratum, Bryol. Europ. Hylocom. Monogr. p. 6, t. 2.

$\mathrm{H}_{A B}$. ad terram, in abietinis montium editiorum.

421. Hypnum OAKesiI.

Sulliv. Icon. Musc. p. 159, t. 102.

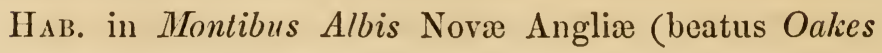
detexit), rarissimum. 
422. Hypnum Alleghaniense.

C. Mull. - Sulliv. Icon. Musc. p. 161, t. 103.

$\mathrm{H}_{\mathrm{B}}$. ad rupes saxaque irrigua, in umbrosis montanis.

\section{Hypnum Bigelowit.}

Sulliv. in Bot. Pacif. R. R. Survey (Whipple), p. 189, t. 8.

НАв. ad rupes, in præruptis humidis Californiæ (Bigelow, Bolander).

424. HyPNuM MYOSUROIDES.

Linn.-Isothecium myosuroides, Bryol. Europ. Isothec. Monogr.p. 7, t. 2.

$\mathrm{H}_{\text {AB. }}$ in sylvis montanis ad rupes.

\section{HYPNUM STOLONIFERUM.}

Hook. Musc. Exot. t. 74.

$\mathrm{H}_{\mathrm{AB}}$. in truncis sylvarum Californiæ (Bolander comm.).

426. Hypnum Brewerianum.

Lesqx. in Trans. Amer. Phil. Soc. 13, p. 12.

НАв. in rupibus graniticis planitiei Californix (Brewer, Bolander).

427. Hypuun Breweriayum, var.

Cæspitibus laxioribus sordide luteis, ramis elongatis quandoque flagelliferis; foliis perichætialibus breviùs acuminatis dentatis; capsula longiore subcernua; 
peristomii interni ciliis perforatis, ciliolis interpositis gracilioribus. An species propria?

Haв. in præruptis profundis Californiæ, ad truncos (Bolander legit).

428. Hypnum hians.

Hedw. - Sulliv. Icon. Musc. p. 163, t. 104.

HAB. ad terram nudam in sylvaticis.

429. Hypnum PILIFERUM.

Schreb.-Eurhynchium piliferum, Bryol.Europ.Eurhyn. Monogr.p. 16, t. 13.

HАв. in graminosis umbrosis (James, C. H. Peck).

\section{Hypnum SullivantiI.}

R. Spruce. - Sulliv. Icon. Musc. p. 165, t. 105.

НАB. ad terram argillosam, secus ripas rivulorum sylvaticas.

431. HypNum STRIgOSUM.

Hoffm. - Eurhynchium strigosum, Bryol. Europ. Eurhyn. Monogr. p. 2, t. 1.

НАв. in argillosis declivis sylvaticis.

432. HYPNUM DIVERSIFOLIUM.

Schimp. in litt. - Eurhynchium diversifolium, Bryol. Europ. Eurhyn. Monogr. p. 4, t. 2.

$\mathrm{H}_{\mathrm{AB}}$ ad terram, in castanetis arenosis collinis Ohionis inferioris. 
433. Hypnum StockesiI.

Turn. - Eurhynchium Stockesii, Bryol. Europ. Eurhyn. Monogr. p. 10, t. 8.

НАв. in sylvis dumetisque, ad terram et rupes Californiæ (Bolander comm.).

\section{Hypnum Oreganum.}

Sulliv. in Musc. U. S. S. Pacif. Expl. Exped. (Wilkes), p. 16, t. 13. Hypnum Douglasii, Hook. MSS., ex Millen in Proced. Linn. Soc. (1864), p. 36.

$\mathrm{HAB}_{\mathrm{A}}$. in truncis putridis sylvarum Californiæ inferioris (Bolander comm.).

435. Hypnum Boscir.

Schwagr. - Sulliv. Icon. Musc. p. 167, t. 106. HАв. ad terram rupesque in collibus umbrosis.

436. Hypnum serrulatum.

Hedw. - Sulliv. Icon. Musc. p. 169, t. 107.

$\mathrm{H}_{\mathrm{AB}}$. ad terram in sylvis.

437. HYPNUM DEPRESSUM.

Bruch. - Rhynchostegium depressum, Bryol. Europ. Rhynchos. Monogr. p. 8, t. 6 .

Hab. ad terram umbrosam secus ripas fluminis Schuylkill, Pennsylvaniæ (James legit).

438. Hypnum deplanatum.

Schimp. - Sulliv. Icon. Musc. p. 171, t. 108.

$\mathrm{H}_{\mathrm{AB}}$. in sylvis ad terram argillosam, raro fructificat. 
439. Hy' PNUM RUSCIFORME.

Weis. - Rhynchostegium rusciforme, Bryol. Europ. Rhyn. Monogr. p. 11, t. 9.

НАB. ad saxa lignumque in rivulis montanis.

440. Hypnum demissum.

Wils. - Raphidostegium demissum, Bryol. Europ. Raphid. Monogr. p. 4, t. 1.

$\mathrm{H}_{\mathrm{AB}}$. in rupibus irriguis, per montes Alleghanienses præprimis meridionales.

441. Hypnum demissum, var.

H. Carolinianum, C. Mull. Synop. Musc. 2, p. 327.

$\mathrm{H}_{\mathrm{AB}}$. ad margines rivulorum Georgiæ et Carolinarum.

442. Hypnum demissum, var.

H. Marylandicum, C. Mull. Synop. Musc. II. p. 328.

$\mathrm{H}_{\mathrm{AB}}$. in iisdem locis antecedentis, plerumque subfluitans.

443. Hypnum microcarpun.

C. Mull. - Sulliv. Icon. Musc. p. 175, t. 110.

$\mathrm{H}_{\mathrm{AB}}$. in arborum cortice ab Ohione usque ad Floridam, montosa fugiens.

444. Hypnum microcarpon, var.

Capsula inclinata. - Hypnum admistum, Sulliv. Musc. Cub. Wright. in Proceed. Amer. Acad. 5, p. 289 (1861).

НА в. ad rupes madidas Ohionis inferioris et Novæboracensis, sat frequens. 
445. Hypnum cylindricarpun.

C. Mull. - Sulliv. Icon. Musc. p. 173, t. 109.

$H_{A B}$ ad truncos putridos in sylvis profundis.

\section{Hypnum recurvans.}

Schwagr. - Sulliv. Icon. Musc. p. 177, t. 111.

$\mathrm{H}_{A B}$. in montibus editioribus ad terram truncosque emortuos, fiequens.

\section{Hypuum ReCurvans, var.}

НАB. in iisdem locis cum præcedente.

\section{Hypyum albulum.}

C. Mull. - Sulliv. Icon. Musc. p. 179, t. 112.

HAB. ad humum truncosque emortuos in montosis.

\section{Hypnum albulum, var.}

HAB. ad terram humidam, secus ursorum semitas, in jugis Black Mountain Carolinæ Septentrionalis.

\section{HypNum EUGYRIUM.}

W. P. Schimp. - Limnobium eugyrium, Bryol. Europ. Limn. Monogr. Suppl. I. p. 1, t. 1.

HẠB. ad rupes madidas in Montibus Albis Novæ Angliæ (legit Oakes).

\section{HypNun MOLLE.}

Dicks. - Limnobium molle, Bryol. Europ. Limn. Monogr.p. $5, t$. 3 et 4 .

НАв, in rivulis montanis Carolinæ Superioris. 
452. Hypnum ochraceum.

Turn. - Limnobium ochraceum, Bryol. Europ. Limn. Monogr. Suppl. 1, p. 2, t. Suppl. 2.

$\mathrm{H}_{\mathrm{AB}}$ ad rupes madidas in alpinis Novæ Angliæ (legit Oakes).

453. Hypnum montanum.

Wils. - Sulliv. Icon. Musc. p. 181, t. 113.

$\mathrm{H}_{\mathrm{AB}}$. in montibus editioribus ad rupes irriguas (Oakes primus legit).

454. Hypnum cuspidatum.

Linn. - Bryol. Europ. Hyp. Monogr. p. 51, t. 36.

$\mathrm{H}_{\mathrm{AB}}$. in graminosis paludosis, sterile.

\section{Hypnum SCHREberi.}

Willd. - Bryol. Europ. Hyp. Monogr. p. 51, t. 37.

$\mathrm{H}_{\mathrm{AB}}$. in montanis humidiusculis ad terram, vulgare.

456. Hypnum Schreberi, var.

$\mathrm{HAB}_{\mathrm{A}}$. in alpibus Novæ Angliæ (legit beatus Oakes).

457. Hypnum cordifolium.

Hedw. - Bryol. Europ. Hyp. Monogr. p. 47, t. 32.

НАв. in turfosis et paludosis septentrionalibus.

\section{Hypnum giganteUm.}

Schimp. Syn. Musc. Europ. p. 642.

Нав. in uliginosis Pennsylvaniæ (Porter), et fossis paludosis Wisconsin. 
459. Hypnum SCORPIOIDES.

Linn. - Bryol. Europ. Hyp. MIonogr. p. 44, t. 30.

$\mathrm{H}_{\mathrm{AB}}$. in uliginosis Ohionis, sterile.

460. Hypnum stranineun.

Dicks. - Bryol. Europ. Hyp. Monogr. p. 49, t. 34.

$\mathrm{H}_{\mathrm{AB}}$. in turfosis montanis septentrionalibus.

461. Hypnum sarmentosum.

Wahl. - Bryol. Europ. Hyp. Monogr. p. 48, t. 33.

H. stramineum, var. Musc. Amer. Exsicc. No. 311 , $E d .1$.

$\mathrm{H}_{\mathrm{AB}}$. in turfosis editioribus Novæ Angliæ (Oakes).

462. Hypnum trifariUm.

Web. et Mohr. - Bryol. Europ. Hyp. Monogr. p. 50, t. 35 .

$\mathrm{H}_{\mathrm{AB}}$. in turfosis profundis Ohionis, sterile.

463. Hypnum uncinatum.

Hedw. - Bryol. Europ. Hyp. Monogr. p. 31, t. 20.

$\mathrm{H}_{\mathrm{AB}}$. in sylvis montanis, ad saxa truncosque putridos.

464. Hypnum Revolvens.

Swartz. - Bryol. Europ. Hyp. Monogr. p. 32, t. 21. $\mathrm{H}_{\mathrm{AB}}$. in uliginosis Ohionis septentrionalis, sterile.

\section{- 465. Hypnum fluitans.}

Linn. - Bryol. Europ. Hyp. Monogr. 1) 34, t. 22. $\mathrm{H}_{\mathrm{AB}}$. in fossis uliginosis, sterile. 
466. Hrpnum ADUNCUM.

Hedw. - Bryol. Europ. Hyp. Monogr. p. 35, t. 24.

НАв. in pratis uliginosis, Ohionis.

\section{Hypnum aduncum.}

Var. gracilescens, Bryol. Europ. Hyp. Monogr. l. $c$. НАв. in fontibus calcariis Pennsylvaniæ.

\section{Hypnum aduncum.}

Var. giganteun, Bryol. Europ. $l$. $c$.

$\mathrm{H}_{\mathrm{AB}}$, in stagnantibus Minnesotæ et Noræ Angliæ montosæ, sterile.

\section{Hypnum filicinum.}

Linn. - Bryol. Europ. Hyp. Monogr. p. 40, t. 27. $\mathrm{HAB}_{\mathrm{A}}$ ad terram calcariam in fontibus.

\section{Hypnum Crista-Castrensis.}

Linn. - Bryol. Europ. Hyp. Monogr. p. 30, t. 19.

$\mathrm{HAB}_{\mathrm{A}}$ in pinetis montanis ad terram.

\section{Hypnum molluscum.}

Hedw. - Bryol. Europ. Hyp. Monogr. p. 29, t. 18.

$\mathrm{H}_{\mathrm{AB}}$ ad terram saxaque in sylvis montanis.

\section{HyPNum CUPRESSIFORME.}

Linn. - Bryol. Europ. Hyp. Monogr. p. 25, t. 14 et 15. HAB. in sylvis editioribus, ad saxa arbores truncosque putridos. 
473. HrPNuM CUPRESSIFORMe, rar.

HАв. in montibus Noгæ Angliæ (Oakes).

474. HypNum CIRCINALE.

Hook. Musc. Exot. t. 107.

НАв. ad corticem arborum in sylris Californiæ montanæ (Bolander comm.).

475. HYPNUM IMPONENS.

Hedw. - Bryol. Europ. Hyp. Monogr. p. 28, t. 17.

$\mathrm{H}_{\mathrm{AB}}$. in truncis deciduis sylrarum, vulgare.

476. HypNum subinponens.

Lesqx. in Trans. Amer. Phil. Soc. 13, p. 14.

Stereodon plumifer, Mitten in Proceed. Linn. Soc. (1864), p. 41.

HАB. ad rupes umbrosas Californiæ inferioris, secus ripas (Bolander legit).

477. HYPNUM REPTILE.

Michx. - Bryol. Europ. Hyp. Monogr. p. 18, t. 7.

$\mathrm{H}_{\mathrm{AB}}$, in montanis ad arbores, vulgatissimum.

478. HypNum hanulosum?

Bryol. Europ. Hyp. Monogr. p. 20, t. 10.

HaB. per montes Noræ Angliæ in truncis putridis (Oakes, James). 
479. Hypnum Fertile.

Sendt. - Bryol. Europ. Hyp. Monogr.p. 22, t. 11.

$\mathrm{H}_{\mathrm{AB}}$. in iisdem locis ac præcedens (James), et in sylvis profundis montium Adirondack.

480. Hypnum curvifolium.

Hedw. - Sulliv. Icon. Musc. p. 183, t. 114.

$\mathrm{H}_{\mathrm{AB}}$ ad truncos prostratos in sylvis, vulgare.

481. Hypnum curvifolium, var.

НАB. ad truncos putridos in paludosis sylvaticis.

482. Hypnum Haldanianum.

Grev. - Bryol. Europ. Hyp. Monogr. p. 23, t. 12.

$\mathrm{HAB}_{\mathrm{A}}$ ad humum truncosque prostratos in sylvis montanis.

483. Hypnum nemorosum.

Koch._Bryol. Europ. Hyp. Monogr. p. 24, t. 13.

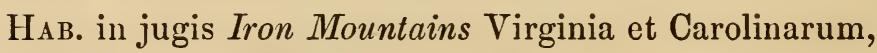
ad lignum cariosum.

484. Hypnum PRATENSE.

Koch.-Bryol. Europ. Hyp. Monogr. p. 43, t. 29.

HAB. per montes Novæ Angliæ (Oakes).

485. Hypnum RUGOSUn.

Ehrh. - Bryol. Europ. Hyp. Monogr. p. 41, t. 28.

$\mathrm{H}_{\mathrm{AB}}$. in locis apricis præsertim calcariis. 
486. HYPNUM NiteNs.

Schreb. - Bryol. Europ. Hyp. Monogr. p. 53, t. 39.

НАв. in turfosis septentrionalibus.

\section{HypuUm SALEBROSUM.}

Hoffm. - Brachythecium salebrosum, Bryol. Europ. Brachyth. Monogr.p. 16, t. 15 et 16.

НАв. in sylvis montanis ad truncos prostratos.

\section{HYPNUM LeTUM.}

Brid. - Sulliv. Icon. Musc. p. 185, t. 115.

HAB. in sylvis ad truncos prostratros, vulgare.

489. HypNum Letum, var.

HАB. in sylvis siccioribus.

490. HypNuM Letum, var.

$\mathrm{H}_{\mathrm{AB}}$. in sylvaticis, ad terram arborumque radices.

491. Hypnum acuminatum.

Beauv. - Sulliv. Icon. Musc. p. 187, t. 116.

$\mathrm{HAB}_{\mathrm{AB}}$ ad truncos emortuos in sylvis, haud rarum.

492. Hypnum aCUminatum.

Var. Rupincolum. - Leskea rupincola, Hedw. Sp. Musc.

p. 227, t. 54 .

НАв. ad terram rupesque sylvarum. 
493. Hypnum acuminatum.

Var. setosum. - Leskea setosa, Hedw. Sp. Musc. p. $226, t .57$.

НАв. in truncis prostratis.

\section{Hypnum RUtabulum.}

Linn. - Brachy thecium rutabulum, Bryol. Europ. Brachyth. Monogr. p. 11, t. 9 et 10.

$\mathrm{H}_{\mathrm{AB}}$, in umbrosis humidis ad saxa.

\section{Hypnum Rutabulum, var.}

НАB. ad terram in umbrosis et humidis, rarius.

496. Hypnum Plumosum.

Linn. - Brachy thecium plumosum, Bryol. Europ. Brachyth. Monogr. p. 4, t. 3.

НАв. ad rupes irriguas in rivulis montanis.

497. Hypnum plumosum.

Var. homomallum, Bryol. Europ. l. c.

НАв. in locis similibus Carolinæ Superioris montosæ.

498. Hypnum plumosum, var.

$\mathrm{HAB}_{\mathrm{A}}$ ad saxa in rivulis montosis Carolinarum.

499. Hypnun Populeum.

Hedw. - Brachythecium populeum, Bryol. Europ. Brachyth. Monogr. p. 3, t. 1 et 2.

НАв. ad saxa erratica Ohionis; etiam per montes Novæ Angliæ. 
500. Hypnum velutinum.

Linn. - Brachythecium velutinum, Bryol. Europ. Brachyth. Monogr. p. 5, t. 4.

$\mathrm{H}_{\text {AB. }}$ in sylvis et umbrosis per montes Novanglos et Noræboracenses (Frost, Peck).

501. Hypnum Fenderer.

Sulliv. Icon. Musc. p. 189, t. 117.

Hab. juxta Santa Fé Neo-Mexicanorum (Fendier).

502. Hypnum Bolanderi.

Lesqx. in Trans. Amer. Phil. Soc. 13, p. 12.

$\mathrm{H}_{\mathrm{AB}}$. ad terram, in dumetis sub umbram Oreodaphnis Californica (Bolander legit et comm.).

503. Hypnum RefLexuir.

Starke. - Brachythecium reflexum, Bryol. Europ. Brachyth. Monogr.p. 8, t. 5.

НАв. in montibus Novæ Angliæ (Oakes); etiam in Pennsylvania montosa.

\section{Hypnum Starkit.}

Brid.-Brachythecium Starkii, Bryol. Europ. Brachyth. Monogr. p. 10, t. 7 .

$\mathrm{H}_{\mathrm{AB}}$. in Nova Anglia montosa (Oakes).

505. Hypnum Rivulare.

Bruch. - Brachythecium rirulare, Bryol. Europ. Brachyth. Monogr. p. 13, t. 12.

Hab. ad rupes irriguas in montibus Pennsylranix. 
506. Hypnum vallium (n. $s p$. )

Hypno læto inflorescentia statura foliatione et habitu accedens ; distinctissimum autem caule rigidiore, pedicello scabro, foliorum cellulis quadratis ad basis angulos late decurrentes paucioribus haud granulosis, etc.

$\mathrm{H}_{\mathrm{AB}}$ ad rupes schistosas in profundis convallibus Californiæ (Bolander).

507. Hypnum Nove Anglie.

Sulliv. \& Lesqx. - Sulliv. Icon. Musc. p. 191, t. 118.

Hab. per montes Novæ Angliæ (beat. Oakes primus legit).

508. Hypnum ILLECEBRUM.

Schwagr. - Scleropodium illecebrum, Bryol. Europ. Sclerop. Monogr. p. 3, t. 2.

$\mathrm{H}_{\mathrm{AB}}$. in graminosis et umbrosis, ad terram et rupes juxta San Francisco Californiæ, vulgare (Bolander legit).

509. Hypnum illecebrum, var.

Caulis divisionibus subdendroideis, foliis subintegerrimis, etc.

HAB. ad rupes in convallibus profundis et umbrosis (Cañons) Californiæ (Bolander legit).

\section{Hypnum CÆSPITOSUM.}

Wils. -Scleropodium cæspitosum, Bryol. Europ. Sclerop. Monogr.p. 2, t. 1.

HAB. ad terram umbrosam et rupes humidas Californix inferioris, vulgare (Bolander legit). 


\section{Hypnum Californicum.}

Lesqx. in Trans. Amer. Phil. Soc. 13, p. 13.

$\mathrm{H}_{\mathrm{AB}}$. in solo arido et arenis umbrosis Californiæ (Bolander legit).

\section{HypNum ARENARIUN.}

Lesqx. in Trans. Amer. Phil. Soc. 13, p. 13.

HAB. in arenis umbrosis juxta San Francisco, Californiæ (Bolander).

\section{Hypnun Pinnatifidui (n. $s p$. )}

Ab Hypno aureo affinissimo distinguitur capsula cernua, annulo majore, foliis brevius filiformi-acuminatis, reti multo laxiore, denique inflorescentia revera dioica.

НАв, in umbrosis vallibus Californiæ (Bolander).

\section{Hypnum Nuttallit.}

Wils. - Sulliv. Icon. Musc. p. 211, t. 128.

Leskea Californica, Hampe in Linnæa (1859), p. 460. Нав. in California (Bigelow, Bolander).

515. Hypnum stellatum.

Schreb. - Bryol. Europ. Hyp. Monogr. p. 14, t. 4. НАв. in pratis humidis Ohionis.

516. HYPNUM POLYMORPHUM.

Hook. \& Tayl. - Bryol. Europ. Hyp. Monogr. p. 13, t. 3.

$\mathrm{H}_{\mathrm{AB}}$. in sabulosis ad radices arborum. 
517. HyPNUM POLYMORPHUM.

Var. MINUS.

Hab. ad truncos putridos Thuje occidentalis, per montes Novæboracenses.

518. Hypnum hispidulum.

Brid. - Sulliv. Icon. Musc. p. 193, t. 119.

$\mathrm{H}_{\mathrm{AB}}$ ad radices arborum in siccioribus.

519. Hypnum subTile.

Hoffm. - Amblystegium subtile, Bryol. Europ. Amblyst. Monogr. p. 4, t. 1 .

Haв. ad radices salicum, circa stagnum Fresh Pond dictum, prope Boston Novæ Angliæ.

520. Hypnum minutissinum.

Sulliv. \& Lesqx. - Sulliv. Icon. Musc. p. 195, t. 120. $\mathrm{H}_{\mathrm{AB}}$. in umbrosis humidis ad lapides, infrequens.

521. Hypnum adnatum.

Hedw. - Sulliv. Icon. Musc. p. 197, t. 121.

$\mathrm{H}_{\mathrm{AB}}$. in umbrosis ad saxa, rarius arboreum.

522. Hypnum adnatum, var.

Ramis gracilioribus, longioribus, foliis densius imbricatis, etc.

НАв. in rupibus arenaceis irriguis Ohionis inferioris. 
523. Hypnum serpens.

Linn. - Amblystegium serpens, Bryol. Europ. Amblyst. Monogr. p. 9, t. 3.

НАв. ad truncos decompositos in sylvis, rarum.

\section{Hypnum radicale.}

Brid.-Amblystegium radicale, Bryol. Europ. Amblyst. Monogr.p. 10, t. 4.

$\mathrm{HAB}_{\mathrm{A}}$ in ligno putrido sylvarum, vulgare.

525. Hypuun Radicale, var.

HАB. ad margines fossarum in ligno putrido.

526. Hypnun ORThOCLADON.

Beauv. - Sulliv. Icon. Musc. p. 199, t. 122.

$\mathrm{HAB}_{\mathrm{B}}$ ad saxa terramque in humidiusculis.

\section{HyPNuM RIPARIUM.}

Linn. - Amblystegium riparium, Bryol. Europ. Amblyst. Monogr. p. 14, t. 8.

НАв. ad saxa lignaque, in stagnantibus atque rivulis.

\section{HyPNun RIPARIUM, var.}

Caulibus abbreviatis, foliis minoribus, capsula breviori. $\mathrm{HAB}_{\mathrm{AB}}$ in iisdem locis antecedentis.

\section{HYPNUM LESCURII.}

Sulliv. Icon. Musc. p. 203, t. 124.

HАв. ad rupes irroratas cataractæ Tallulah Falls dictæ in Georgia montosa ; etiam in Pennsylvania (invenit cl. Porter). 
530. HypNum PULChELLUM.

Dicks. - Plagiothecium pulchellum, Bryol. Europ. Plag. Monogr. p. 9, t. 4.

$\mathrm{H}_{\triangle \mathrm{B}}$. in montibus Noranglis (James), et Noræboracensibus.

\section{Hypnum Denticulatum.}

Dill. - Plagiothecium denticulatum, Bryol. Europ. Plag. Monogr. p. 12, t. 8.

$\mathrm{H}_{\mathrm{AB}}$. in sylvis montanis, ad ligna putrida terramque umbrosam.

532. Hypnum denticulatum, var.

$\mathrm{H}_{\mathrm{AB}}$. in montibus, ad terram.

533. HyPNUM MUHLENBECKII.

Br. et Sch. - Plagiothecium Muhlenbeckii, Bryol. Europ. Plag. Monog. p. 11, t. 6.

$\mathrm{H}_{\mathrm{A}}$. in Nova Anglia montosa (Oakes).

\section{Hypnum sylvaticun.}

Brid._Plagiothecium sylvaticum, Bryol. Europ. Plag. Monogr. p. 14, t. 11.

Нав. in Montibus Albis Novæ Angliæ (Oakes).

535. Hypnum Sullivantie.

Schimp. - Sulliv. Icon. Musc. p. 207, t. 126.

$\mathrm{H}_{\mathrm{AB}}$. in rupium fissuris terra impletis Ohionis et Novæ Angliæ. 
MUSCI BOREALI-AMERICANI.

536. HYPNUM WRightiI.

Sulliv. Icon. Musc. p. 209, t. 127.

Omalia Wrightii, Sulliv. in Musc. Exsicc. No. 269, Ed. 1.

Hab. ad radices Querci virentis circa San Antonio Texanum (Wright); prope Santa Fé Neo-Mexicanum (Fendler). 



\section{N D E X.}

Acaulon muticum, C. Mull. Schimperianum, Sulliv. triquetrum, C. Mull.

Alsia abietina, Hook. Californica, Sulliv. longipes, Sulliv. et Lesqx.

Amblyodon dealbatus, Beauv. Amblystegium radicale, Bryol. Europ. riparium, Bryol. Europ. serpens, Bryol. Europ. subtile, Bryol. Europ.

Anacalypta Starkeana, Hedw.

Anacamptodon splachnoides, Brid. 379

Andræa crassinervia, Bruch, 26 petrophila, Ehrh.

Rothii, Web. et Mohr, rupestris, Hedw.

rupestris, Turn.

Anomodon apiculatus, Bryol.

\section{Europ.}

attenuatus, Hartm. obtusifolins, Bryol, Europ.

? Toccoæ, Sulliv, et Lesqx.

? tristis, Cesati, viticulosus, Hook. et Tayl.

Antitrichia Californica, Sulliv. curtipendula, Brid. curtipendula, var.

A phanorherma serratum, Sulliv. 356

Archidium Olioense, Bryol, Europ. 35

Arctoa fulvella, Bryol. Europ.

Astomum nitidulum, Bryol. Europ. 47 Sullivantii, Bryol. Europ.

Atrichum angustatum, Hook. crispum, James, undulatum, Beauv.

Aulacomnium androgynum Schwæor.

heterosticluum, Bryol. Furop. 309 palustre, Schwægr. turgidum, Schwægr.

Barbula amplexa, Lesqx. artocarpa, Lesqx. Bolanderi, Lespx.
No.
Barbula brachyphylla, Sulliv. caspitosa, Schwægr. cirrhata Bryol. Europ. convoluta, Hedw. allax, var. Bryol. Europ. $\quad 129$ flexifolia, Hampe, 132 inermis, Mont. 142 lævipila, Bryol. Europ. $\quad 143$ latifolia, Bryol. Europ. $\quad 144$ membranifolia, Schultz, $\quad 126$ mucronifolia, Schwægr. $\quad 141$ Mulleri, Bryol. Europ. $\quad 147$ papillosa, Wils. $\quad \mathbf{1 2 7}$ rigida, Schultz, $\quad 125$ ruralis, Hedw. $\quad \mathbf{1 4 5}$ ruralis, var. $\quad 146$ tortuosa, Web. et Mohr, 137 unguiculata, Hedw. $\quad 128$ vinealis, Brid. 130 vinealis, var. 131 virescens, Lesqx. 134

Bartramia calcarea, Bryol. Europ. 250 conostoma. Bryol. Europ. $\quad 261$ fontana, Brid. $\quad 251$ fontana, var. $\quad 252$ ithyphylla, Brid. $\quad 255$ Marchica. Schwogr. $\quad 253$ Menziezii, Turu. $\quad 259$ Menziezii, var. $\quad 260$ Muhlenbergii, Schwægr. $\quad 25 \%$ Oederi, Swartz, 258 pomiformis, Hediv. $\quad 257$ radicalis, Beauv. 254 stricta, Brid. 256

Blindia acuta, Bryol. Furop. $\quad 113$ Brachythecium Plumosum, Bryol. Europ. populeum, Bryol. Europ. $\quad 449$ reflexum, Bryol. Europ. $\quad 503$ rivulare, Bryol. Eurrop. 505 rutabulum, Bryol. Europ. 494 salebrosum, Bryol. Europ. $\quad 4 \$ 7$ Starkii, Bryol. Europ. $\quad 504$ velutinum, Bryol. Europ. 500 Braunia Californica, Lesqx. 226 
Bruchia brevifolia, Sulliv. brevipes, Honk.

flexuosa, C. Mull.

flexuosa, var.

Ravenelii, Wils.

Bryum acuminatum, Hoppe et Hornsch.

alpinum, Linn.

annotinum, Hedw.

argenteum, Lin.

atropurpureum, Web. et Mohr,

bimum, Schreb.

cæspiticium, Linn.

cæspiticium, var.

Californicum, Sulliv.

capillare, Hedw.

cernuum, Hedw.

crudum, Schreb.

cncullatum, Schwægr.

Duvalii, Voit.

elongatum, Dicks.

intermedium, Brid.

Lescurianum, Sulliv.

Ludwigii, Spreng.

nutans, Sclireb.

nutans, var.

44

45

41

42

43

264

293

271

290

291

278

288

289

292

285

262

269

265

286

266

277

273

obconicum, Hornsch.

occidentale, Sulliv.

occidentale, var.

pallescens, Schwægr.

pseudo-triquetrum, Schwægr. 281 pyriforme, Hedw.

roseum, Schreb.

torquescens, Bryol. Europ.

Tozeri, Grev.

turbinatum, Hedw.

uliginosum, Brid.

Wahlenbergii, Schwægr.

Wahlenbergii, var.

Buxbaumia aphylla, Haller,

Campylopus Leanus, Sulliv.

(cesqux. 9

Tallulensis, Sulliv. et Lesqx.

viridis, Sulliv, et Lesqx.

Campylostelium saxicola, Bryol. Europ.

Catoscopium nigritum, Brid.

Ceratodon purpureus, Brid. purpureus, var.

276

282

272

287

263

274

275

331

94

93

Cryphæa glomerata, Schimp.

Clasmatodon parvulus, Hampe, parvulus, var.

Climacium Americanım, Bricl. Conomitrium Julianum, Mont.

Conostomum boreale, Swartz,

Coscinodon Wriglıtii, Sulliv.

347

368

369

402

112

261

197

Cylindrotliecium brevisetum, Wils. 39

cladorrhizans, Hedw. compressum, Hedw.

386
Cylindrothecium Drummondi, Bryol. Europ.

seductrix, Hedw.

Sullivantii, C. Mull.

387

389

Desmatodon arenaceus, Sulliv. et Lesqx.

latifolius. Brid.

Neo-Mexicanus, Sulliv et

Lesqx.

124

nervosus, Bryol. Europ. 121

plinthobius, Sulliv. et Lesqx. 123

Dichelyma capillaceum, Dill. $\quad 345$ falcatum, Myrin, $\quad 343$

pallescens, Bryol. Europ. $\quad 346$

Swartzii, Schimp. 344

Dicranodontium longirostre, Web. et Mohr,

Dicranum Blyttii, Bryol. Europ. 69 cerviculatum, Hedw. $\quad 65$

condensalum, Hedw. $\quad 86$

congestum, Brid. $\quad 80$

debile, $\mathrm{H}$ et $\mathrm{W}$. 62

Drummondi, C. Mull. $\quad \delta 8$

elongatum, Schwægr. $\quad 79$

flagellare, Hedw. $\quad \mathbf{7 2}$

gracilescens, Web. et Mohr, 57

heteromallum, Hedw. $\quad 67$

heteromallum, var. $\quad 68$

interruptum, Bryol. Europ. $\quad 73$

longifolium, Hedw. $\quad \mathbf{7 4}$

montanum, Hedw. 71

Muhlenbeckii, Bryol. Europ. 81 pallidum, C. Mull. 77 pallidum, Bryol. Europ. $\quad 85$

palustre, Brid. 83

pellucidum, Hedw. $\quad 60$

polycarpum, Ehrh. $\quad 58$

rhabdocarpum, Sulliv. $\quad 82$

robustum, Bryol. Europ. $\quad 88$

rufescens, Turn. $\quad 64$

Schraderi, Schwægr. $\quad 84$

Schreberi, Hedw. $\quad 61$

scoparum, Hedw. 75

scoparium, var. $\quad 76$

scoparium, var. $\quad \mathbf{7 7}$

scoparium, var. $\quad$ is

spurium, Hedw. $\quad 85$

spurium, var. $\quad 86$

Starkii, Web. et Mohr, $\quad 70$

subulatum, Hedw. $\quad 66$

undulatum, Turn. $\quad 87$

varium, Hedw. $\quad 63$

virens, Hedw. 59

Didymodon cylindricus, N. et $\mathrm{H} .158$ rubellus, Bryol. Europ. $\quad 157$

Diphysicum foliosum, Web. et Mohr,

Distichium, capillaceum, Bryol. Europ.

332

388 inclinatum, Bryol. Europ.

161

162 
INDEX .

Drummondia clavellata, Hook.

Encalypta ciliata, Hedw. rhabdocarpa, Sch wægr. vulgaris, Bryol. Furop.

Entosthodon Bolanderi, Lesqx. Drummondi, Sulliv. Templetonii, Schwægr.

Ephemerum cohærens, Hampe, crassinerviım, Schwægr. synoicum, James,

Eurhynchium diversifolium, Bryol. Europ. piliferum, Bryol. Europ. Stockesii, Bryol. Europ. strigosum, Bryol. Europ.

Eustichium Norvegicum, Bryol. Europ.

Fabronia gymnostoma, Sulliv. et Lesqx.

pusilla, Raddi,

Ravenelii, Sulliv.

Ravenelii, var. Wrightii, Sulliv.

Fissidens adiantoides, Hedw. bryoides, Hedw. exiguus, Sulliv grandifrons, Brid. incurvus, Schwægr. limbatus, Sulliv. minutulus, Suiliv. obtusifolius, Wils. osmundoides, Hedw. polypodioides, Hedw. Ravenelii, Sulliv. subbasilaris, Hedw. taxifolius, Hedw.

Fontinalis antipyretica, Lin. antipyretica, var. antipyretica, var. biformis, Sulliv. biformis, var. Sulliv. Dalecarlica Bryol. Europ. disticha, H. et W.

Lescurii, Sulliv.

Lescurii, var.

Neo-Mexicana, Sulliv.

Novæ Angliæ, Sulliv.

Funaria Californica, Sulliv. et Lesqx.

flavicans, Michx. hygrometrica, Hedw. hygrometrica, var. serrata, Brid.

Grimmia Californica, Sulliv.

Californica, var. calyptrata, Hook. Donniana, Smith, leucophæa, Grev. montana, Bryol. Europ. Olneyi, Sulliv.
194 Grimmia ovata, Web. et Mohr,

213

214

210

204

207

208

trichopliylla, Grev.

trichophylla, var.

Gymnostomum curvirostrum, Hedw.

curvirostrum, var. rupestre, Schwægr. rupestre, var.

432 Hedwigia ciliata, Ehrh. ciliata, var.

133

433

431

63

163

378

375

376

377

374

108

103

100

111

104

105

101

99

109

110

102

107

106

333

334

335

337

338

342

339

340

341

334

336

238

240

241

242

239

205

206

211

216

212

215

209

Homalothecium subcapillatum, Bryol. Europ.

Hookeria acutifolia? Hook.

Hylocomium brevirostre, Bryol. Europ.

splendens, Bryol. Europ.

squarrosum, Bryol. Europ. $\quad 416$

triquetrum, Bryjol. Europ. $\quad 417$

umbratum, Bryol. Europ. $\quad 420$

Hymenostomum microstomum, $\mathrm{R}$.

Brown.

Hypuum abietinum, Linn. $\quad 413$

acuminatum, Beauv. 491

acuminatum, var. $\quad 492$

acuminatum, var. $\quad 493$

admistum, Sulliv. $\quad 444$

adnatum, Hedw. $\quad 521$

adnatum, var. $\quad 522$

aduncum, Hedw. $\quad 466$

aduncum, var. $\quad 46 \pi$

aduncum, var. $\quad \mathbf{4 6 8}$

albulum, C. Mull. $\quad 448$

albulum, var. $\quad 449$

arenarium, Lesqx. $\quad 512$

Alleghaniense, C. Mull. $\quad 422$

Blandowii, Web. et Mohr, 414

Bigelowii, Sulliv. $\quad 423$

Bolanderi, Lesqx. $\quad 502$

Boscii, Schwægr. $\quad 435$

brevirostre, Ehrh. $\quad 418$

Brewerianum, Lesqx. $\quad 426$

Brewerianum, var. $\quad 427$

Carolinianum, C. Mull. $\quad 441$

cæspitosum, Wils. 510

Californicum, Lesqx. $\quad 511$

circinale, Hook. $\quad 474$

cordifolium, Hedw. $\quad \mathbf{4 5 7}$

crispifolium, Hook. 405

Crista-Castrensis, Linn. $\quad 470$

cupressiforme, Linn. 472

cupressiforme, var. $\quad 473$

curvifolium, Hedw. $\quad 480$

curvifolium, var. $\quad 481$

cuspidatum, Linn. $\quad 454$

cylindrocarpum, C. Mull. $\quad \mathbf{4 4 5}$

delicatulum, Linn. $\quad 404$

demissum, Wils. $\quad 440$

2

1

5

84


Hypnum demissum, var.

demissum, var.

denticulatum, Dill.

denticulatum, var.

deplanatum, Schimp.

depressum, Bruch,

diversifolium, Schimp.

Douglasii, Honk.

eugyrium, Schimp.

Fendleri, Sulliv.

fertile, Sendt.

filicinum, Linn.

fluitans, Linn.

giganteum, Schimp.

gracile, Br. et Sch.

gracile, var.

gracile, var.

liamulosum, Bryol. Europ.

Haldanianum, Grev.

hians, Hedw.

hispidulum, Brid.

illecebrum, Schwægr.

illecebrum, var.

imponens, Hedw.

lætum, Brid.

lætum, var.

lætum, var.

Lescurii, Sulliv.

leuconeuron, Sulliv. et

$$
\text { Lesqx. }
$$

Marylandicum, C. Mull. microcarpum, C. Mull.

microcarpum, var.

minutissimum, Sulliv.

minutulum, Hedw.

molle, Dicks.

molluscum, Hedw.

montanum, Wils.

Muhlenbeckii, Br. et Sch.

myosuroides, Linn.

nemorosum, Koch,

nitens, Schreb.

Novæ Angliæ, Sulliv. et Lesqx.

Nuttallii, Wils.

Oakesii, Sulliv.

ochraceum, Turn.

Oreganum, Sulliv.

orthocladon, Beauv.

paludosum, Sulliv.

piliferum, Schreb.

pinnatifidum, Sulliv. et

Lesqx.

plumosum, Linn.

plumosum, var.

plumosiun, var.

polymorphum, Hook.

polymorphum, var.

populeum, Hedw.

pratense, Koch,
441 Hypnum pulchellum, Dicks. $\quad 530$

442 pygmæum, Br. et Sch. $\quad 408$

531 radicale,Brid. 524

532 radicale, var. 525

438 recurvans, Schwægr. 446

437 recurvans, var. 447

432 reflexum, Starke, 503

434 reptile, Michx. 477

45ı) revolvens, Swartz, 464

501 riparium, Linn. 527

479 riparium, var. 528

469 rivulare, Bruch, 505

465 rugosum, Ehrh. 485

458 rusciforme, Weis, 439

410 rutabulum, Linn. 494

411 rutabulum, var. 495

412 salebrosum, Hoffm.

478 sarmentosum, Wahl. 461

$482 \quad$ Schreberi, Willd. 455

428 S.chreberi, var. 456

518 scitum, Beauv. 409

508 scorpioides, Linn. 459

509 serpens, Linn. 523

475 serrulatum, Hedw. 436

488 splendens, Hedw. 419

489 squarrosum, Linn. 416

490 Starkii, Brid. 504

529 stellatum, Schreb. 515

stoloniferum, Hook. 425

stramineum, Dicks. $\quad 460$

stramineum, var. $\quad 461$

strigosum, Hoffm. $\quad 431$

subimponens, Lesqx. $\quad 476$

subtile, Hoffm. 519

Sullivantiæ, Schimp. $\quad 535$

Sullivantii, R. Spruce, $\quad 430$

sylvaticum, Brid. $\quad 534$

tamariscinum, Hedw. $\quad 403$

trifarium, Web. et Mohr, 462

triquetrum, Linn. $\quad 417$

triste, C. Mull.

umbratum, Ehrh. $\quad 420$

uncinatum, Hedw. $\quad 463$

vallium, Sulliv. et Lesqx. $\quad \mathbf{5 0 6}$

velutinum, Linn. $\quad 500$

Whippleanum, Sulliv. $\quad 407$

Wrightii, Sulliv. 536

434

526

415

429

513

496

497

498

516

517

499

484

Isothecium myosurvides, Bryol. Europ.

Leptodon Ohioense, Sulliv. $\quad 354$ trichomitrion, Mohr, $\quad 352$

trichomitrion, var. $\quad 353$

Leskea Californica, Hampe, 514 denticulata, Sulliv. $\quad 367$ fragilis, $H$. et $W$. 363 obscura, Hedw. 365 polycarpa, Hedw. $\quad 364$ rostrata, Hedw. $\quad 366$ rupincola, Ilediv. $\quad 492$ 
INDEX.

Leskea setos $\pi$, Herliv.

Leucobryuin minus, Hampe, vulgare, Hampe,

Leucodon brachypus, Brid. julacens, Hedw.

Limnobium eugyrium, Bryol. Europ. molle, Bryol. Europ. ochraceum, Bryol. Europ.

Macromitrium Dregei, Hornsch.

Meesia longiseta, Hedw. longiseta, var. tristicha, Funk, uliginosa, Hedw. uliginosa, var.

Mnium affine, Bland. affine, var. affine, var. cinclidioides, Huben. cuspidatum, Hedw. Drummondi, Br. et Sch. hornum, Hedw. lycopodioides, Hook. Menziesii, Hook. punctatum, Hedw. serratum, Brid. spinulosum, Bryol. Europ. stellare, Hedw.

Myurella Careyana, Sulliv.

Neckera chlorocaulis, C. Mull. Douglasii, Honk. complanata, Hedw. Menziesi, Hook. oligocarpa, Bryol. Europ. pennata, Hedw.

Octodiceras Julianum. Brid.

Omalia Jamesii, Schimp. Wrightii, Sulliv.

Oncophorus glaucus, Bryol Europ. Orthotrichuin affine, Schrad. anomalum, Hedw. Canadense, Br. et Sch. crispulum, Hornsch. crispum, Hedw. cupulatum, Hoffm. cylindrocarpum, Lesqx. Ḧutchinsiæ, H. et. 'T. leiocarpum, Bryol. Europ. Lyellii, Hook.

Lyellii, var.

Ludwigii, Schwægr. obtusifolium, Schrad. psilocarpum, James, Rogeri? Brid. speciosum, Nees, strangulatum, Beauv. Sturmii, H. et $H$. Texanum, Sulliv.

Phascum cuspidatum, Schreb. pritens, Hedw.

Phyllogonium Norvegicum, Brid.
Physcomitrella patens, Bryol. Europ.

Physcomitrium pyriforme, Bryol. Europ.

immersum, Sulliv.

234

233

Plagiothecium denticulatum, Bryol. Europ.

Muhlenbeckii, Bryol. Europ. 533 pulchellum, Bryol. Europ. $\quad 530$ sylvaticum, Bryol. Europ. $\quad 534$

Platygyrium repeus, Bryol. Europ.

Pleuridium alternifolium, Brid. alternifolium, var. 37 alternifolium, var. $\quad 38$ palustre, Bryol. Europ. $\quad 40$ subulatum, Bryol. Europ. $\quad 39$

Pogonatum alpinum, Brid. $\quad 320$ alpinum, var. 321 brachyphyllum, Beauv. $\quad 317$ brevicaule, Brid. $\quad 316$ capillare, Brid. $\quad 318$ urnigerum, Brid. $\quad 319$

Polytrichadelphus Lyallii, Mitten, 322

Polytricluum commune, Linn. $\quad 329$ commune, var. 330 formosum, Hedw. $\quad 323$ gracile, Menz. $\quad 324$ juniperinum, Hedw. $\quad 327$ juniperinuın, var. $\quad 328$ piliferum, Sclireb. $\quad 325$ piliferum, var. $\quad 326$

Pottia Heimii, Bryol. Europ. $\quad 115$ minutula, Schwægr. 117 subsessilis, Schwægr. $\quad 118$ truncata, Hedw. 116

Pterigyuandrum filiforme, Hedw. 348

Pterogonium gracile, Swartz, 349 Ptychomitrium Drummondi, Wils. 196 incurvum, Schwægr. 195

Pylaisæa intricata, Bryol. Europ. 380 Jamesii, Sulliv. et Lesqx. $\quad 383$ subdenticulata, Sulliv, $\quad 382$ velutina, Bryol. Europ. $\quad 381$

Racomitrium aciculare, Brid. $\quad 217$ canescens, Brid. 221 canescens, var. $\quad 222$ fasciculare, Brid. $\quad 220$ lanuginosum, Brid. $\quad 223$ microcarpon, Brid. $\quad 219$ Sudeticum, Brid. 218

Rapidostegium demissum, Bryol. Europ.

Rhabdoweisia denticulata, Bryol. Europ. fugax, Bryol. Europ.

Rhynchostegium depressum, Bryol. Europ. rusciforme, Bryol. Europ. 
Schistidinm Agassizii, Sulliv. et Lesqx. apocarpum, Hedw. apocarpum, var. apocarpum, var. confertum, Funk, confertum, var.

Schlotheimia Sullivantii, C. Mull. 192 Scleropodium illecebrum, Bryol. Europ. cæspitosum, Bryol. Europ.

Sphagnum acutifolium, Ehrh. acutifolium, var. auriculatum, Schimp. cuspilatum, Ehrh. cuspidatum, var. cuspidatum, var. cyclophyllum, Sulliv, et Lesqx. cymbifolium, Ehrh. fimbriatum, Wils. humile, W.T. Schimp. Lescurii, Sulliv. macrophyllum, Bernh. molle, Sulliv. molluscum, Bruch. Mulleri, Schimp. Portoricense Hampe. Pylaisii, Brid. Pylaisii, var. recurvum, Beauv. rigidum, Schimp. rigidum, var. sedoides, Brid. subsecundum, Nees. subsecundum, var. subsecundum, Sullivantianum Austin, squarrosum, Pers.
Sphagnum Torreyanum, Sulliv. $\quad 11$

Splachnum ampullaceum, Linn. 230

Stereodon plumifer, Mitten, $\quad \mathbf{4 7 6}$

Syrrhopodon Floridanus, Sulliv. 168

T'etraphis pellucida, Hedw. $\quad 164$

Tetraplodon angustatus, Bryol. Europ. australis, Sulliv, et Lesqx. $\quad 227$ mnioides, Hedw. 229

Thelia asprella, Sulliv. $\quad 370$ hirtella, Sulliv. 371 Lescurii, Sulliv. $\quad 372$

Thuidium abietinum, Bvyol. Europ. 413 Blandowii, Bryol. Europ. $\quad 414$ delicatulum, Bryol Europ. $\quad 404$ minutulum, Bryol. Europ. $\quad 406$ tamariscinum, Bryol. Europ. 403

Timmia Austriaca, Hedw. $\quad 312$ megapolitana, Hedw. $\quad 311$

Trematodon ambiguus, Bryol.
Europ. longicollis, Michx. 95

Trichostomum flexipes, Bryol.
Europ. fragile, Hook. 149

glaucescens, Hedw. $\quad 156$ pallidum, Hedw. 155 rigidulum, Smith, $\quad 150$ tophaceum, Brid. $\quad 151$ tortile, Schrad. 152 tortile, var. 153 vaginans, Sulliv. 154

Weisia cirrhata, Hedw. $\quad 48$ viridula, Brid. $\quad 49$

Zygodon Californicus, Hampe, 172 Lapponicus, Hedw. 169 Mougeotii, Bryol. Europ. $\quad 170$ Sullivantii, C. Mull. $\quad 169$ 
in

$+1$$$
\text { - }
$$

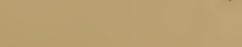




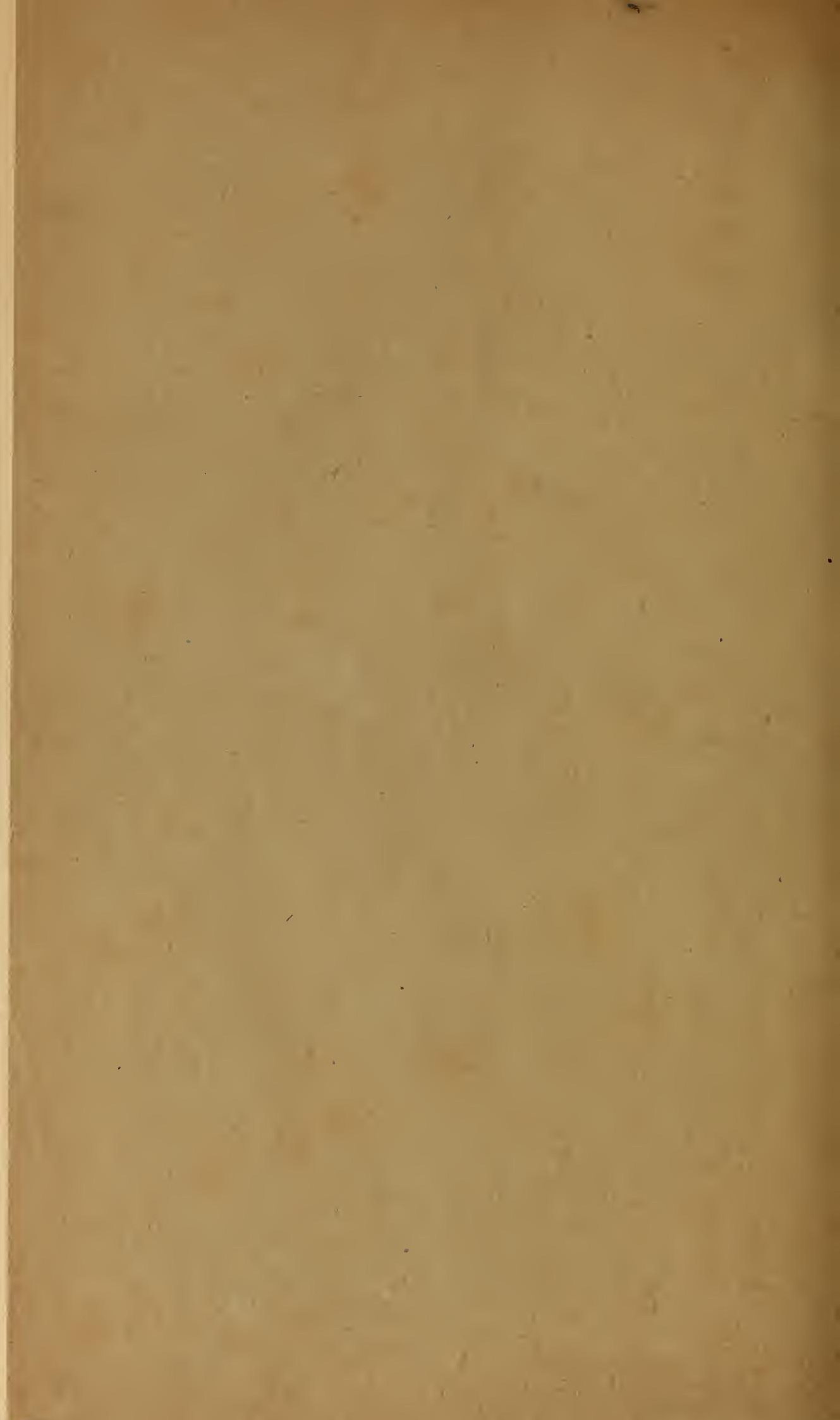







\section{QK541.S91 1865 gen \\ Sullivant, William/Musci boreali-america}

I I I ' I

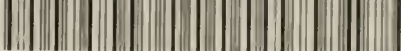

35185000015865 
